\title{
Basic coding activities of populations of Xenopus laevis olfactory receptor neurons recorded with a fast confocal line illumination microscope
}

\author{
Dissertation \\ for the award of the degree \\ "Doctor rerum naturalium" \\ submitted by \\ Mihai Alevra \\ born in \\ Bukarest, Romania
}

\begin{abstract}
Division of Mathematics and Natural Sciences
of the Georg-August-University Göttingen
\end{abstract}

Göttingen, 2012 
Prof. Dr. Dr. Detlev Schild (Reviewer) Abtl. Neurophysiologie und Zelluläre Biophysik Zentrum Physiologie und Pathophysiologie

Prof. Dr. Tim Salditt (Reviewer) Institut für Röntgenphysik

Prof. Dr. Christoph Schmidt Abtl. Molekulare und Zelluläre Biophysik III. Physikalisches Institut 
Herewith I declare, that I prepared the PhD Thesis 'Basic coding activities of populations of Xenopus laevis olfactory receptor neurons recorded with a fast confocal line illumination microscope' on my own and with no other sources and aids than quoted. 


\section{Contents}

1 Introduction $\quad 1$

1.1 Olfaction . . . . . . . . . . . . . . . . . 1

1.1.1 Organization of the main olfactory system . . . . . 2

1.1.2 Transduction . . . . . . . . . . . . . . 3

1.1.3 Temporal coding .............. 5

1.1.4 Aims .................... 5

2 Materials and Methods $\quad 7$

2.1 Microscopy . . . . . . . . . . . . . . . . 7

2.1.1 Modifications of the LIM . . . . . . . . . 7

2.2 Image processing . . . . . . . . . . . . . . . 9

2.2.1 Shift correction . . . . . . . . . . . . . 9

2.2.2 Neighborhood correlation map . . . . . . . . . . . 10

2.2.3 Activity correlation imaging (ACI) . . . . . . . . 10

2.3 Xenopus laevis tissue slice preparation and staining . . . . . . 11

2.4 Perfusion and stimulus application . . . . . . . . . . . 12

2.5 Electrophysiology . . . . . . . . . . . . . . . . . . 12

2.6 X-Ray phase contrast microscopy . . . . . . . . . . . . 13

3 Results $\quad 15$

3.1 ORN population responses . . . . . . . . . . . . . 15

3.1.1 Evaluation of single-spike detection using fluo-5F . . . 16

3.1 .2 Imaging protocol . . . . . . . . . . . . . . 18

3.1.3 Shift correction and ROI selection . . . . . . . . . . 19

3.1.4 ORN population responses to a single odor . . . . . . . 19 
3.1.5 Correction of photobleaching effect . . . . . . . . 21

3.1.6 Distribution and concentration dependency of response

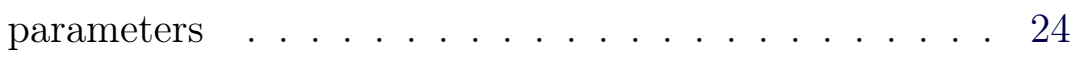

3.2 Calcium ion influx in single ORNs . . . . . . . . . . . . 26

3.2.1 Fast confocal imaging in frame mode $($ ytz $) \ldots 28$

3.2.2 Sequential line imaging (tyz) . . . . . . . . . . . . 29

3.2.3 Analysis of 4D data using model-driven ACI . . . . . . 31

3.2.4 Pixel-wise fit of time traces . . . . . . . . . . . . . 34

3.2.5 Distribution of VGCCs in ORN somata and total calcium ion current . . . . . . . . . . . . . . 37

3.3 Effect of BK channel blocker iberiotoxin . . . . . . . . . . . 39

3.4 X-Ray phase contrast microscopy . . . . . . . . . . . . . . . . 41

4 Discussion $\quad 43$

4.1 Conclusions . . . . . . . . . . . . . . . . . . 43

4.2 ORN population measurements . . . . . . . . . . . . 44

4.2 .1 Onset latencies . . . . . . . . . . . . . . . . . 45

4.2.2 Action potential frequencies . . . . . . . . . . 46

4.2 .3 Role of glomeruli . . . . . . . . . . . . . . . . . . 46

4.3 Calcium channel microdomains and function of BK channels . 47

$\begin{array}{lll}5 & \text { Summary } & 49\end{array}$

6 Bibliography $\quad 51$ 


\section{Chapter 1}

\section{Introduction}

\subsection{Olfaction}

A plethora of different substances diffuse through the biosphere, many of them being produced by living organisms and eventually released into their surrounding space. The resulting concentrations depend on the distance to the source and the molecular composition can be highly specific to the underlying metabolism. This is a pool of information animals can use to their advantage, by the detection and discrimination of molecules using the sense of smell - olfaction.

Most animals depend on olfaction to survive. They need to find food, avoid toxins and predators, locate mates and recognize parents or offspring. It is remarkable how well the olfactory system performs on all these tasks, considering that it has to detect and interpret an immense number of possible molecules with a limited number of neurons, while still being able to discriminate between similar odors.

The main strategy in olfactory sensing becomes evident from its general organization, which is introduced in the next section. Other properties, however, as the temporal patterns of neuronal activation and how they relate to the olfactory stimulus, are not as well understood and need more refined measurements on different scales. 


\subsubsection{Organization of the main olfactory system}

The vertebrate olfactory system mainly consists of three parts, the olfactory epithelium (OE) located in the nasal cavity containing the olfactory receptor neurons (ORN), the olfactory bulb (OB) where the ORN activity is first processed, and higher brain centers (Fig. 1.1).

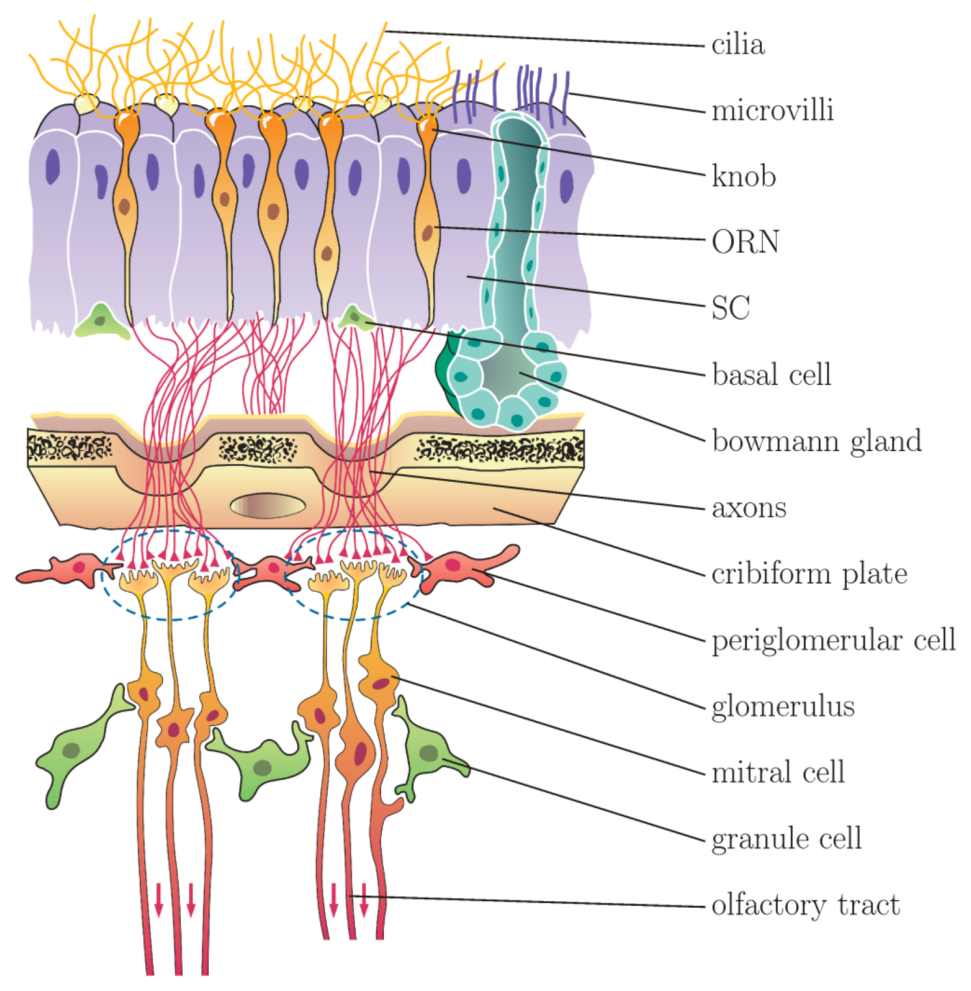

Figure 1.1: Overview of the vertebrate olfactory system. The main aspects of olfactory coding discussed in this thesis concern the activity of the olfactory receptor neurons (ORN), their projections into different glomeruli, and the activity of mitral cells. Image modified from [17, 29].

The olfactory receptor neurons are bipolar neurons with a dendrite extending from their soma towards the lumen of the nasal cavity and an axon projecting into the OB. The dendrite is terminated by a so-called dendritic knob from which cilia or microvilli protude into the mucus, which covers the contact area between $\mathrm{OE}$ and the outer environment and is generated by sus- 
tentacular cells and by the bowman's glands [15, 18, 62, 63]. Odor molecules are detected by specific olfactory receptors (OR) in the ciliary membrane (see 1.1.2), potentially leading to a depolarization of ORNs upon binding and action potentials traversing the unmyelinated axon along the olfactory nerve towards the OB. There, groups of ORN axons converge into separate neuropil structures called olfactory glomeruli, forming glutamatergic synaptic connections between ORN axon terminals and dendrites from groups of mitral/tufted $(\mathrm{M} / \mathrm{T})$ cells $[3,12,41,42]$.

The convergence pattern of ORN projections into glomeruli is determined by the ORN specificity. By typically expressing one type ${ }^{1}$ of OR from a large set of OR genes (about 1000 and 350 in mice and humans, respectively [4, 45, $74,76]$ ), ORNs have specific sensitivities to a range of different odors. Most ORNs expressing the same OR are found to project to the same glomerulus $[53,71]$.

This is a major aspect in the olfactory system. By sorting ensemble responses based on classes of odor sensitivity, the high-dimensional response of all ORNs is abstracted, compressed, and signal-amplified. Odors in the OB are thus represented by their glomerular (and $\mathrm{M} / \mathrm{T}$ cell) response pattern, which is an efficient way to sample a large odor space with few neurons. Usually this is referred to as "combinatorial coding" [33]. The glomerular response patterns have been recently successfully investigated by $\left[\mathrm{Ca}^{2+}\right]_{i}$ imaging in a statical way $[35,46]$, in which odors are characterized by assigning each glomerulus a single number that represents its respective response strength.

However, patterns of activation might change over time in an odor-specific way, as the transduction of the signals in ORNs can have various time courses. This is illustrated in the next section.

\subsubsection{Transduction}

Olfactory Receptors are located on cilia of ORNs (Fig. 1.2) and coupled to olfactory $G$ Proteins $\left(\mathrm{G}_{\text {olf }}[21]\right)$. Upon binding of an odorant molecule, the

\footnotetext{
${ }^{1}$ The concept of the expression of a singular OR per ORN [20, 52] has been recently challenged [17, 49, 68].
} 


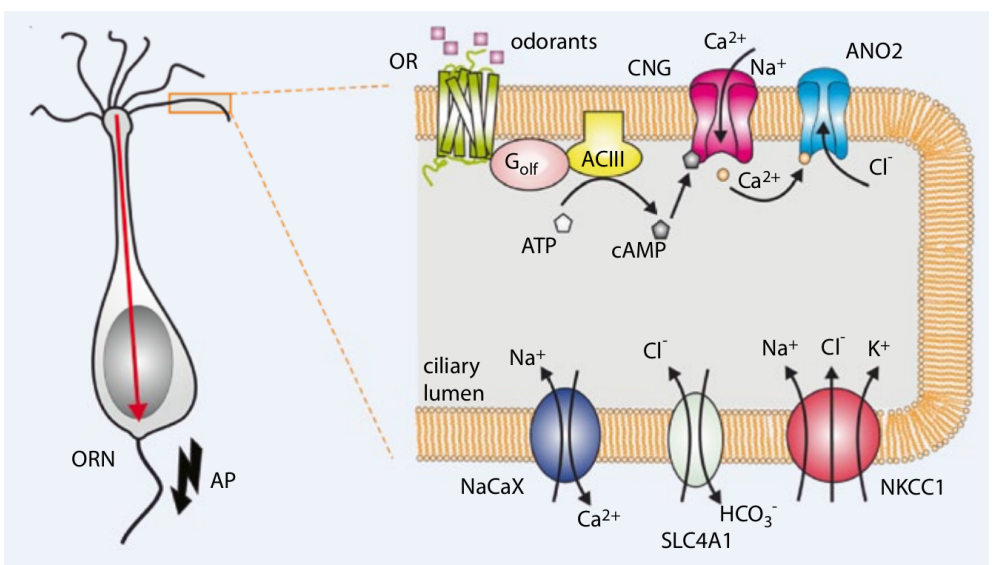

Figure 1.2: Olfactory transduction for the cAMP pathway. [36].

$\alpha$-subunit of $\mathrm{G}_{\text {olf }}$ activates adenylyl cyclase type III (ACIII), which in turn generates cAMP from ATP. Subsequently, nucleotide-gated (CNG) cation channels are opened upon cAMP binding [8], eliciting mostly a depolarizing $\mathrm{Ca}^{2+}$ current into the cell. In turn, $\mathrm{Ca}^{2+}$ dependent $\mathrm{Cl}^{-}$channels (ANO2) are opened after binding of intracellular $\mathrm{Ca}^{2+}$, further depolarizing the cilium through $\mathrm{Cl}^{-}$outflux [67]. Additionally, $\left[\mathrm{Ca}^{2+}\right]_{i}$ is reported to inhibit $\mathrm{CNG}$ currents [27], facilitating adaptation.

If the combined ciliary currents are sufficient to depolarize the soma through electrotonic propagation (red arrow in Fig. 1.2) above the opening threshold of somatic voltage-gated $\mathrm{Na}^{+}$channels, action potentials are generated, further depolarizing the cell. This in turn also activates voltagegated $\mathrm{Ca}^{2+}$ channels and subsequently $\mathrm{Ca}^{2+}$ dependent $\mathrm{K}^{+}$channels [59], which have been shown to modulate neuronal activity in several ways [57].

From the multitude of interconnected reactions in the transduction pathway described above, it is clear that the ORN response in the form of action potentials over time might depend on odor concentration and other factors (like the history of previous states) in a complex manner, and that its description by a one-dimensional dose-response curve could be too simplistic. 


\subsubsection{Temporal coding}

Fast measurements of $\mathrm{M} / \mathrm{T}$ cell activity have recently revealed that temporal patterns are indeed very important for olfactory coding [23]. Here, the most significant information was found in the vector of first-spike latencies. By comparing the order of latencies (using rank-correlation) from different $\mathrm{M} / \mathrm{T}$ cells for individual odor applications, the odor identity (and, to a lesser degree, the odor concentration) could be reliably predicted. As a comparison, the pattern of mean firing rates reconstructed from the same data showed much less predictive power.

Surprisingly, the information for odor identity was already contained in the first $400 \mathrm{~ms}$, at a time when the statical response pattern had not fully developed. This is supported by results from behavioral studies in various species, where discrimination tasks showed that $500 \mathrm{~ms}$ or less were already sufficient for odor recognition [1, 28, 54, 64, 69, 72].

A possible explanation lies in the relatively broad OR specificity. Similar odors could activate almost the same set of ORNs at a longer time scale, and therefore be difficult to be distinguished with static response patterns, while first-spike latencies would profit from odor-dependent information contained in fast ORN transduction kinetics. The necessary data to test such hypotheses does however not exist.

\subsubsection{Aims}

This thesis aims at eludicating ORN population coding and to relate it to the latency coding observed in the olfactory bulb. How are $\mathrm{M} / \mathrm{T}$ latencies generated? Does latency coding exist already at ORN level? What is the actual overlap in static OB response patterns that could make them less efficient at odor coding than temporal response patterns? Unfortunately, these questions concern a large range of scales in space and time. No known technique could possibly measure fast ORN activity while at the same time record complete static response patterns in the OB. This thesis is therefore divided into three parts, each focusing on one spatiotemporal scale using different techniques, with the aim of combining the results for a better understanding 
of temporal olfactory coding.

1. Fast measurement of ORN populations. Responses of ORN populations are to be recorded with highest possible temporal resolution using $\left[\mathrm{Ca}^{2+}\right]_{i}$ imaging. A typical simple odor should be chosen that is able to stimulate more than one ORN class at the same time. Temporal properties of the responsive ORNs should be determined and analyzed on population level and their possible contribution to latency coding evaluated.

2. $\left[\mathrm{Ca}^{2+}\right]_{i}$ dynamics in single ORNs. As $\left[\mathrm{Ca}^{2+}\right]_{i}$ might not directly reflect ORN activity due to different sources of $\mathrm{Ca}^{2+}[30]$, fast $\left[\mathrm{Ca}^{2+}\right]_{i}$ imaging should be used in combination with the patch-clamp technique to evaluate the contribution of VGCCs and therefore the validity of $\left[\mathrm{Ca}^{2+}\right]_{i}$ imaging in part 1 .

3. X-Ray Microscopy of full nose-brain preparations. This technique is to be evaluated for the possibility to image populations of ORNs and their projections in the OB simultaneously and in 3D. The long-term aim is to evaluate the overlap of static OB response patterns for different olfactory stimuli. 


\section{Chapter 2}

\section{Materials and Methods}

\subsection{Microscopy}

For fast confocal fluorescence imaging a custom line illumination microscope (LIM) was used. The LIM consists of an upright microscope frame (Axioscope FS 2 plus, Zeiss, Göttingen) in combination with a confocal unit (Fig. 2.1) designed and built by Dr. Stephan Junek [22]. My contribution was to develop the software for microscope control (scan mirror position, position of the objective, triggers for laser shutter, camera synchronization and external events) and the software for image acquisition.

\subsubsection{Modifications of the LIM}

During the time of this thesis, the LIM was modified as follows:

Excitation laser and laser shutter The argon ion excitation laser was replaced due to increasing fluctuation in output power by an optically pumped semiconductor laser (Sapphire LP 488, Coherent, Santa Clara USA). A custom laser shutter was build using the coil of a hard drive (similar to [32]).

CMOS line detector Because of electrical oscillatory noise, the CCD line camera (1x512 pixels) was replaced by a fast CMOS line camera (2x2048 pixels) capable of fast readout (Sprint spL2048-140km, Basler AG, Ahrensburg, 

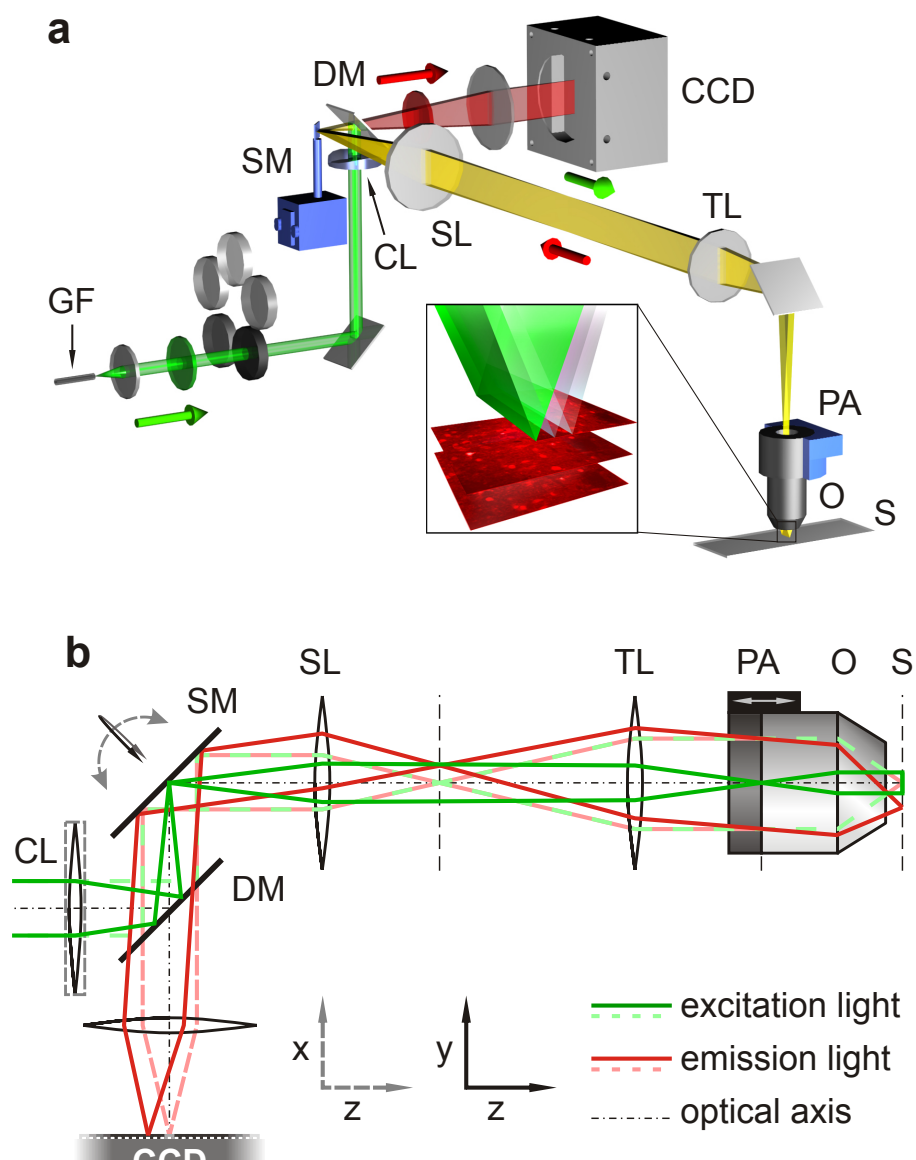

CCD

Figure 2.1: Light pathway in the LIM. The emission laser light exiting the glass fibre (GF) is collimated and then focussed onto the scan mirror (SM) by a cylindrical lens (CL) after being reflected by the dichroic mirror (DM). A horizontal illumination line is imaged onto in the back aperture of the objective $(\mathrm{O})$ by the scan lens (SL) and tube lens (TL), its angle to the optical axis defined by the deflection angle of the SM. A vertical illumination line is thus created in the object plane (S) with a horizontal displacement depending on SM deflection angle and its axial position defined by the piezo actuator (PA) of the objective. Emission light is descanned by the SM, passes through the DM and an additional emission filter, and is imaged by the detector lens onto the linear detector, which also has the function of a confocal aperture. Image taken from [22]. 
Germany). The emission pathway was adjusted to the new detector and pixel size. The image acquisition software was newly written in $\mathrm{C}++$ as the driver architecture of the grabber card changed. Unfortunately, it was discovered that the baseline dark readout for each line fluctuates between time points. To compensate for this effect, the sensor was covered from emission light for a region of 128 pixels, and the average of this dark signal subtracted from individual pixel counts for each time point during acquisition, which significantly reduced image noise. A similar approach named dynamic baseline clamp is used in recent CMOS cameras (e.g. Neo sCMOS, Andor, Belfast, $\mathrm{UK})$.

\section{$2.2 \quad$ Image processing}

\subsubsection{Shift correction}

To detect a shift [26] between two similar images $f(x)$ and $g(x)$ (here in 1-D), their non-normalized cross-correlation function

$$
c c_{f, g}(\Delta x)=\int_{-\infty}^{+\infty} f(x) \cdot g(x+\Delta x) d x
$$

is searched for the position $x_{0}$ of its maximum. A calculation of $c c_{f, g}$ becomes prohibitively slow for large $2 \mathrm{D}$ or $3 \mathrm{D}$ images and long timeseries. As $c c_{f, g}$ is identical to the convolution $f(x) * g(-x)$, its calculation can be sped up by using the convolution theorem:

$$
f(x) * g(-x)=\mathcal{F}^{-1}\{\mathcal{F}\{f(x)\} \cdot \mathcal{F}\{g(-x)\}\}
$$

with the fourier transform $\mathcal{F}$ and its inverse $\mathcal{F}^{-1}$. For discrete signals of a limited width $d_{x}$, the discrete fast fourier transform is used. However, this assumes that the functions $f(x)$ and $g(x)$ are periodical in $d_{x}$, and their left and right borders are to be correlated. This can introduce errors in the shift detection if objects enter or exit the image area through a border, which is often the case in microscopic data. As a simple way to avoid any border 
interaction, the image data is zero-padded with margins of size $d_{m}$ bigger than the maximum shift, and the corresponding cross-correlation function is corrected by the factor

$$
\frac{1}{d_{x}+d_{m}-\operatorname{abs}(\Delta x)}
$$

to compensate for the contribution that is introduced by correlating two rectangular functions. The resulting correlation function is free of border effects and its maximum position is fitted by a gaussian function for subpixel resolution. This procedure, expanded to $2-\mathrm{D}$ or $3-\mathrm{D}$, accurately determines a shift vector between a reference image (usually a time-average for better signal) and all other images. The shift vector is then used to correct the image shift by cubic spline interpolation of each pixel intensity for its new position.

\subsubsection{Neighborhood correlation map}

As a measure of local correlation, the time signal of a pixel $I_{x, y}(t)$ is correlated with the averaged time signal of its four neighbors $I_{\mathrm{nb}}(t)=\left(I_{x+1, y}(t)+\right.$ $\left.I_{x-1, y}(t)+I_{x, y+1}(t)+I_{x, y-1}(t)\right) / 4$,

$$
c_{\mathrm{nb}}(x, y)=\frac{\sum_{t=1}^{T} \hat{I}_{x, y}(t) \cdot \hat{I}_{\mathrm{nb}}(t)}{\sum_{t=1}^{T} \hat{I}_{x, y}^{2}(t) \sum_{t=1}^{T} \hat{I}_{\mathrm{nb}}^{2}(t)}
$$

where $\hat{I}$ denotes an intensity after subtraction of its average and $T$ is the maximum time index. Maps of $c_{\mathrm{nb}}(x, y)$ can be used to identify connected regions that have similar signals over time.

\subsubsection{Activity correlation imaging (ACI)}

To detect time-correlated pixels between different spatial regions, reference signals $I_{r}(t)$ are obtained by averaging pixel intensities over user-defined pixel regions of interest (ROIs, $r=\mathrm{ROI}$ index) for each time point. The correlation map $c c(x, y, r)$ for each $r$ is calculated identically to Eq. (2.4) (replacing $I_{\mathrm{nb}}$ with $I_{r}$ ). The set of correlation maps for each $r$ can then be mapped into 
color space using custom defined colors for each $r$, creating a high-contrast color image based on the temporal activity patterns [22]. To improve the contrast for image data under photobleaching conditions, the time traces of each pixel are subtracted by their linear regression curve prior to correlation. In this work, only a single ROI was used and therefore no color mapping was necessary.

\subsection{Xenopus laevis tissue slice preparation and staining}

For an optimal comparison to the measurements referenced in section 1.1.3, which were also performed in our lab, the same model animal, Xenopus laevis, was used.

Tadpoles of Xenopus laevis (stages 51-54, staged after [44]) were immobilized by chilling in a mixture of ice and water for 2 min and sacrificed by decapitation as approved by the Göttingen University Committee for Ethics in Animal Experimentation. A tissue block containing both epithelia, olfactory nerves, and the anterior half of the brain was cut out, glued onto the stage of a vibratome (VT 1200S, Leica Microsystems GmbH, Nussloch, Germany) and immersed in solution (referred to as "bath solution" in the following) containing (in $\mathrm{mM}$ ): $98 \mathrm{NaCl}, 2 \mathrm{KCl}, 1 \mathrm{CaCl}_{2}, 2 \mathrm{MgCl}_{2}, 5$ glucose, 5 sodium pyruvate, 10 Hepes; $230 \mathrm{mOsm} / 1, \mathrm{pH}$ 7.8. The block was horizontally cut through the olfactory mucosae and the dorsal half discarded, obtaining 180-220 $\mu \mathrm{m}$-thick slices after a second horizontal cut [37]. Slices were transferred into custom built microscopy chambers and stabilized with a grid [11].

For staining, fluo-4 AM or fluo-5F AM (Invitrogen, Karlsruhe, Germany) were first dissolved in 20\% Pluronic F-127 in DMSO and diluted in bath solution to reach a final concentration of 5-10 $\mu \mathrm{M}$. The final concentrations of DMSO and Pluronic F-127 did not exceed $0.5 \%$ and $0.1 \%$, respectively. Slices were incubated for 30-45 min in $200 \mu \mathrm{l}$ staining solution, with an addition of MK571 (50 M, Alexis Biochemicals, Lörrach, Germany), a specific inhibitor 
of multidrug resistance-associated proteins to prevent transporter-mediated destaining of the slices $[38,40]$. After incubation, the solution was exchanged with bath solution containing MK571 (50 $\mathrm{\mu M})$ and measured after $30 \mathrm{~min}$.

\subsection{Perfusion and stimulus application}

Slices in the recording chamber were continuously perfused with bath solution by gravity feed through a funnel applicator (modified from [34, 58] for a larger tip diameter). Odors were applied into the funnel using an electronic pipette (HandyStep electronic, Brand, Wertheim, Germany) modified to accept TTL triggers generated by the LIM control computer, and delivering $1 \mathrm{ml}$ solution for $2 \mathrm{~s}$.

As odorant stimuli, either a single amino acid (L-Methionine) or a mixture of 15 amino acids was used (Sigma, Deisenhofen, Germany): L-proline, Lvaline, L-leucine, L-isoleucine, L-methionine, L-glycine, L-alanine, L-serine, L-threonine, L-cysteine, L-arginine, L-lysine, L-histdine, L-tryptophane, Lphenylalanine, diluted in bath solution to concentrations given in the experiments.

\subsection{Electrophysiology}

Patch pipettes with series resistances of 7-11 M $\Omega$ were pulled from borosilicate glass with $1.8 \mathrm{~mm}$ outer diameter (Hilgenberg, Malsfeld, Germany) using a two-stage pipette puller (PC-10, Narishige, Japan). Pipette solutions contained (in mM): TEA-Cl 5, $\mathrm{CsMeSO}_{3} 62, \mathrm{MgCl}_{2}$ 2, HEPES 10, ATP-Mg 2, GTP-Na $0.3 \mathrm{Na}_{2}$-Phosphocreatine 7; pH 7.7-7.8 (adjusted with TEAOH), $230 \mathrm{mOsm} / 1$, and with varying concentrations of EGTA, BAPTA, Fluo-4 and fluo-5F (see Results). Patch-clamp was performed using an EPC7 plus amplifier (HEKA, Lambrecht, Germany) and custom hardware/software used in the lab [60].

After formation of a tight seal (1-5 G $\Omega$ ) and setting the holding potential to $-75 \mathrm{mV}$, ORNs were brought into whole-cell configuration by negative 
pressure, breaking the seal. For fast depolarization pulses synchronized with the CCD detector aquisition, an analog switch (DG419, MAXIM, San Jose, USA) was used in a custom-build circuit to switch the command voltage of the patch-clamp amplifier from the output of the patch-clamp software to a manually adjustable level.

\subsection{X-Ray phase contrast microscopy}

Tadpoles were either stained with Nanogold (Nanoprobes, Yaphank, USA, conjucated with $10 \mathrm{kD}$ dextran and the fluorescent dye Texas Red) in collaboration with Dr. Sebastian Gliem (Neurophysiology and Cellular Biophysics, University of Göttingen) using different staining protocols, or left unstained. The specimen were then fixed with 1,5\% glutaraldehyde and 1,5\% paraformaldehyde in $0.1 \mathrm{M}$ sodium phosphate buffer, $\mathrm{pH} 7.3$ for $3 \mathrm{~h}$ at room temperature and given to Dr. Peter Schwartz (Dep. of Anatomy and Embryology, University of Göttingen) for postfixing for two hours in $2 \%$ osmium tetroxide in $0.1 \mathrm{M}$ sodium phosphate buffer and dehydration in graded ethanol. X-Ray phase contrast microscopy and microtomography was then performed by Martin Krenkel (Institute of X-Ray Physics, University of Göttingen). 


\section{Chapter 3}

\section{Results}

\subsection{ORN population responses}

In this section, the responses of olfactory receptor neurons (ORNs) to olfactory stimuli are investigated with respect to olfactory coding. To assess which properties of ORN responses should be measured in the first place, it is useful to think of how information exits the olfactory epithelium to the next neural cell layer: the only carriers of information are action potentials as a stream of unitary events in time for each ORN. As such, ORN output in response to an olfactory stimulus can be characterized in a limited number of ways:

- action potential frequency

- first-spike latency

- individual spike times (and resulting inter-spike intervals)

In the generally accepted model of combinatorial coding, individual ORNs possess specific sensitivities to a range of different odors, and form the response to an odor as an ensemble. It is therefore desirable to measure the above properties for multiple ORNs in parallel.

An established technique to determine neuronal activity is $\left[\mathrm{Ca}^{2+}\right]_{i}$ imaging, which infers the state of neuronal excitation from changes in its intracellular $\mathrm{Ca}^{2+}$ concentration $\left(\left[\mathrm{Ca}^{2+}\right]_{i}\right)$. This is especially valid if the only 
source of $\left[\mathrm{Ca}^{2+}\right]_{i}$ is an influx through voltage-gated $\mathrm{Ca}^{2+}$ channels (VGCCs) with higher activation threshold potential than the voltage-gated $\mathrm{Na}^{+}$channels that facilitate the depolarization, as an increase in $\left[\mathrm{Ca}^{2+}\right]_{i}$ would always (and only) follow an action potential. In ORNs, however, at least two sources of $\left[\mathrm{Ca}^{2+}\right]_{i}$ exist and may overlap during responses:

1. $\mathrm{Ca}^{2+}$ influx through ciliary transduction channels, of which currently cyclic nucleotide-gated (CNG) and IP3-gated are known

2. $\mathrm{Ca}^{2+}$ influx through somatic high-voltage gated $\mathrm{Ca}^{2+}$ channels upon depolarization above $-30 \mathrm{mV}$ [61]

It is unclear if $\left[\mathrm{Ca}^{2+}\right]_{i}$ imaging is principally able to resolve the contributions of VGCC and thus detect single APs even if the temporal resolution of the measurement would be sufficient.

\subsubsection{Evaluation of single-spike detection using fluo-5F}

In the first sets of experiments it was determined if it is possible to reliably detect somatic fluorescence steps attributable to action potentials.

Slice preparations containing the olfactory epithelia of Xenopus laevis larvae (called "slices" in the following) were stained with the fast $\left[\mathrm{Ca}^{2+}\right]$ sensitive fluorescent dye fluo-5F AM. The custom-built confocal line illumination microscope (LIM, [22]) was used to acquire time series of images from one focal plane at $115 \mathrm{f} / \mathrm{s}$. Fig. 3.1 shows relative fluorescence traces $\left(\Delta F / F_{0}=\left(F-F_{0}\right) / F_{0}\right.$ with initial fluorescence $\left.F_{0}\right)$ from 4 cell somata after a stimulation with odorants (mixture of 15 amino acids at $200 \mu \mathrm{M}$ (see Methods) at $\mathrm{t}=0 \mathrm{~s}$ ). For one of the cells (blue trace), fluorescence steps upon odorant stimulation can be clearly detected. The individual fluorescence steps are related to action potentials, as they have almost identical amplitudes which are reached within one frame $(<8 \mathrm{~ms})$. They occur at long time delays (several seconds) from the stimulus onset and at low frequencies (3-6 Hz). Unfortunately, there were very few cells exhibiting this behavior (4 of 60 in 8 slices, $0-1$ per slice), and thus not suitable for population measurements. For the majority of ORNs reacting to odorants, somatic fluorescence 


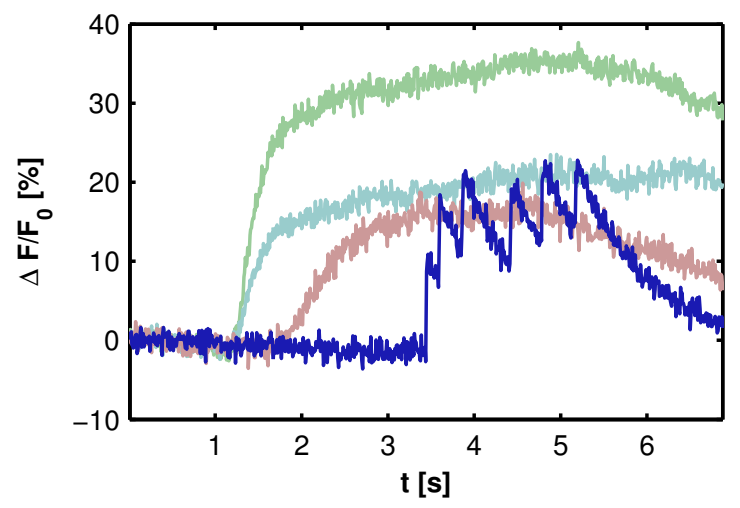

Figure 3.1: Fluorescence steps in a subset of ORNs. Relative fluorescence intensity $\left(\Delta F / F_{0}\right)$ is shown for somatic signals of four cells after application of odorant solution (amino acid mixture, $200 \mathrm{\mu M}$ ). A smooth fluorescence increase (red, cyan, green) is detectable from most ORNs even at frame rates $>100 \mathrm{f} / \mathrm{s}$, with the exception of few cells (blue) showing steep increases ( $<8 \mathrm{~ms}$ total rise time) in fluorescence attributable to action potentials of low frequencies $(3-6 \mathrm{~Hz})$.

increased smoothly. If the odor concentration was reduced 5-fold and the acquisition frame rate was increased to $217 \mathrm{f} / \mathrm{s}$, step-like features could be seen for a small number of cells $(n=8)$. Under all conditions, however, significant photobleaching occurred, quickly reducing response amplitudes and leading to cell death during 1-4 repetitions.

As a conclusion, AP-induced $\mathrm{Ca}^{2+}$ influx through VGCCs was too difficult to detect for population measurements. Fast $\left[\mathrm{Ca}^{2+}\right]_{i}$ dynamics during depolarization are further elucidated in section 3.2. For the measurements on the population level, it was decided to focus on action potential frequencies and onset latencies only, under conditions of lower photobleaching and higher repeatability. The LIM was modified for higher sensitivity (replacing the CCD line detector with a CMOS device), the excitation laser power reduced, and the imaging protocol changed to minimize exposition to the times of odor responses. 


\subsubsection{Imaging protocol}

Slice preparations of olfactory epithelia were stained with the slower but higher-affinity $\left[\mathrm{Ca}^{+2}\right]$ sensitive fluorescence indicator fluo-4 AM $\left(\mathrm{Ca}^{2+}\right.$ dissociation constant $k_{D}=345 \mathrm{nM}[14], k_{D}=2.3 \mu \mathrm{M}$ for fluo-5F) to improve the fluorescence signal at lower $\left[\mathrm{Ca}^{+2}\right]_{i}$. The modified LIM was used to acquire time series of images from one focal plane, using a scanning protocol consisting of 3 phases (Fig. 3.2 C):

1. Slow acquisition: for each image frame, the laser illumination line was scanned over the image area for $10 \mathrm{~ms}$ and then placed for $90 \mathrm{~ms}$ at a "line off" position, where the excitation laser beam did not reach the back aperture of the objective, effectively shutting off the excitation power. The resulting frame rate was $10 \mathrm{f} / \mathrm{s}$ for 40 frames and $4 \mathrm{~s}$, and the image frames were used to determine the initial fluorescence $F_{0}$ as the pixel dwell time was identical to the fast acquisition.

2. Fast acquisition: the image area was continuously scanned at $100 \mathrm{f} / \mathrm{s}$ for 400 frames and $4 \mathrm{~s}$ to capture the ORN responses in high temporal resolution.

3. Slow acquisition: identical to phase 1, for the observation of the slow reduction in $\left[\mathrm{Ca}^{2+}\right]_{i}$ after stimulation, which could last several seconds.

For the olfactory stimulation, odorants were applied towards the end of phase 1 using an electronic pipette to the funnel of the application system. The pipette was triggered by the software for mirror positioning, providing a precise stimulus onset time. To achieve a fast concentration change of the odorant in the imaged region (see Fig. $3.2 \mathrm{D}$ for a calibration using the fluorescent dye fluorescein), the tip of the applicator needed to be placed near the imaged olfactory epithelium $(<750 \mu \mathrm{m})$, which often induced a slight reversible image drift during the application due to variations in flow speed. 


\subsubsection{Shift correction and ROI selection}

A custom algorithm was developed to detect image drifts in time series of $2 \mathrm{D}$ or $3 \mathrm{D}$ images with sub-pixel precision, and was employed to correct any drift during individual measurements (see methods).

ORN fluorescence signals were obtained at their soma, where a region of interest (ROI) was placed and the fluorescence count averaged over all of its pixels for each image frame. A difficulty was the selection of suitable ROIs in the conditions of low signal-to-noise ratio during fast measurements. As a general aid, all pixels were correlated with a step function in time with its edge positioned $2 \mathrm{~s}$ after stimulus onset (where usually all responding cells showed increased fluorescence). In addition, a neighborhood correlation map (see methods) was calculated to localize areas of correlated activity.

The focal plane was placed 5-20 $\mu \mathrm{m}$ below the surface of the slice to image well-stained but healthy ORNs. However, the shape of the surface changed considerably between measurements, unlike in similar experiments in the olfactory bulb or on inverted microscopes (as the glass surface has a stabilizing effect). During 9 repetitions of a measurement, the top cell layer could shrink by $25 \%$, and cell pairs could change their relative positions by over $10 \mu \mathrm{m}$. As these movements represent non-affine transformations in space, they could not be automatically corrected by a simple global algorithm. Instead, the positions of all ROIs were manually adjusted for each measurement, the focal plane adjusted to compensate for shrinking of the slice in the z-direction, and all cells excluded from analysis that moved out of the focal plane.

\subsubsection{ORN population responses to a single odor}

A single amino acid, methionine, was chosen as a prototypic olfactory stimulus, as it is known to elicit responses from a large fraction of ORNs, increasing the average number of cells that can be measured simultaneously. In addition, methionine is recognized throughout multiple ORN "classes" that can be defined by their sensitivity to different odors [39]. This makes it possible to obtain a response vector over a heterogeneous ORN population in the sense of combinatorial coding, which would not be possible with odorant molecules 
to which only one ORN class responds.

To investigate the temporal properties of the ORN responses in respect to stimulus strength, different concentrations of methionine were used: 20, 40 and $80 \mathrm{\mu M}$. These were sufficiently large to cause transient somatic $\left[\mathrm{Ca}^{2+}\right]_{i}$ increase that could be well detected and analyzed, but also sufficiently low to avoid saturation effects for the fluorescent dye or possible adaptation for highly sensitive ORNs [48].

A total of 540 respones from 57 ORNs in 12 slices were successfully recorded, each cell being exposed to 5-12 applications of methionine with alternating concentrations at time intervals of 3 minutes. ROIs containing the cell somata were obtained and adjusted as decribed above, and their fluorescence signal $F_{\text {raw }}$ normalized $\left(F=\left(F_{\text {raw }}-F_{0}\right) / F_{0}\right.$ with the initial fluorescence $F_{0}$ from imaging phase 1$)$. A typical dataset from one ORN is displayed in Fig. 3.2. A map of $F_{0}$ is shown in subimage A, while the (step-) correlation map for ROI selection is depicted in $\mathrm{B}$. In subimage $\mathrm{E}$, multiple fluorecence traces show the typical response approximately $1 \mathrm{~s}$ after stimulus onset (compare to subimage D), color-coded for odor concentration.

Following parameters could be extracted from the individual response traces:

- The maximum amplitude $F_{\max }$ for each trace was obtained by taking the maximum of $F$ after a gaussian blur (using a width of 5 time points $(50 \mathrm{~ms})$ ) for noise reduction.

- The time $t_{0}$ of the response onset was obtained by fitting a piecewise defined exponential function $F_{\text {fit }}$ to the rising phase,

$$
F_{\text {fit }}(t)= \begin{cases}F_{0} & , t<t_{0} \\ F_{0}+a \cdot\left(1-e^{-\frac{t-t_{0}}{\tau}}\right) & , t \geq t_{0}\end{cases}
$$

with fit parameters $t_{0}$, amplitude $a$ and time constant $\tau$. An example fit is given in Fig. 3.2 E as solid blue line. Note, however, that the asymptotic amplitude $a$ is not used as an estimate for the maximum fluorescence amplitude, as the fitted curve does not reflect the post-rise 
part of the response well. A second (falling) exponential component would better describe the whole shape of the response, but needlessly complicate the situation (two additional fit parameters) without giving further insight. Also, only the rising phases of the responses are very similar between different ORNs, while their shape in the post-rise phase can vary considerably, which is difficult to model.

- The slope $S$ of the fitted curve at response onset time $t_{0}$ can be obtained from the time derivative (from positive times) of $F_{\text {fit }}$,

$$
S=\left.\frac{d}{d t} F_{\text {fit }}(t)\right|_{t=t_{0}}=\frac{a}{\tau}
$$

Under the conditions of low $\left[\mathrm{Ca}^{2+}\right]_{i}$ which does not saturate the fluorescent dye (which is fulfilled at $t_{0}$ ) and the main contribution of $\left[\mathrm{Ca}^{2+}\right]_{i}$ increase coming from VGCCs (which can also be assumed near $t_{0}$, as transduction $\mathrm{Ca}^{2+}$ needs time to diffuse to the soma), $S$ can be treated as proportional to the average frequency of action potentials at onset time $t_{0}$ (when referring to the same neuron).

\subsubsection{Correction of photobleaching effect}

Despite the improvements to the imaging conditions, the effect of photobleaching could not be ignored. From the evolution of maximum amplitudes $F_{\text {max }}$ (Fig. 3.3) during 12 odor applications at alternating concentrations, an exponential reduction ( $F_{\max }$ shown as circles) is clearly visible. If amplitudes were to be pooled into sets of equal concentration and averaged, a systematic error would arise from the order of the applications, as later measurements have reduced amplitudes. To correct for this effect, the amplitudes $F_{\max }(n)$ for the application number $n$ were fitted with a single exponential function, using a general decay constant $\tau^{*}$ and three amplitudes $b_{i}(i \in\{1,2,3\}$ for 20, 40 and $80 \mu \mathrm{M}$, respectively) for the three concentrations,

$$
F_{\text {bleach }}(n)=b_{c(n)} \cdot e^{-n / \tau^{*}}
$$




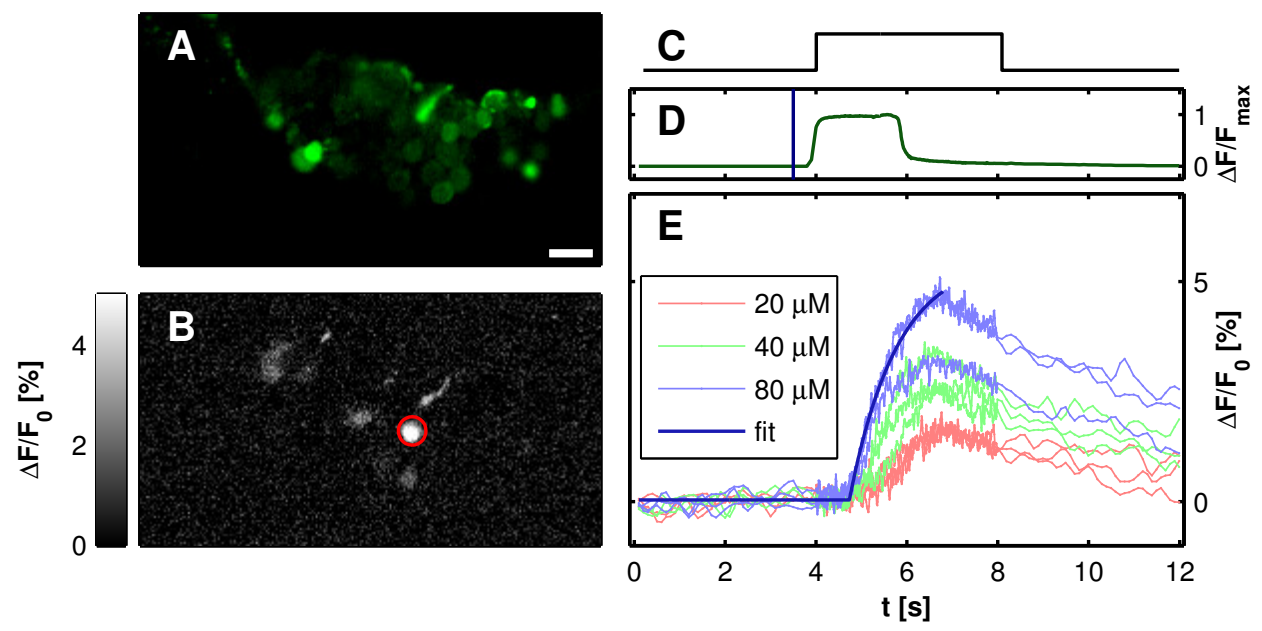

Figure 3.2: Imaging of odor-induced $\left[\mathrm{Ca}^{2+}\right]_{i}$ transients in olfactory receptor neurons. (A) Time-averaged fluorescence intensity from OE slice stained with the $\left[\mathrm{Ca}^{2+}\right]$-sensitive dye fluo-4. Scale bar $20 \mu \mathrm{m}$. (B) Map of the relative fluorescence increase $\Delta F / F_{0}$ calculated from time-averaged signals before (0-4 s) and during (6-8 s) odor responses, respectively. One region of interest (ROI, red) indicates a typical selection of pixels to be averaged for somatic fluorescence traces. (C) Imaging protocol. To reduce photobleaching, the scanning frequency of the galvanometric mirror was increased from 10 to $100 \mathrm{f} / \mathrm{s}$ only for the relevant time range of odor responses (4-8 s). (D) Time course of the odor concentration. The odor stimulus was replaced by the fluorescent dye fluorescein to determine the evolution of stimulus concentration over time (green line) after the electrical trigger (vertical blue line) to the odor application system. The trigger time was adjusted for ORN responses to be in the time frame of fast acquisition without unnecessary photobleaching. (E) Responses of a single ORN (see B) to odorant applications (methionine at different concentrations, see legend), with best-fit curve for the onset of one response shown as thick blue line. 
where the information about what concentration index is used on application $n$ is given in $c(n)$. Using this scheme, the decay constant $\tau^{*}$ was obtained and could be used for photobleaching correction:

$$
F_{\max }^{*}(n)=F_{\max }(n) \cdot e^{n / \tau^{*}}
$$

As the fluorescence slopes $S(n)$ for application $n$ would be similarly influenced by photobleaching, they were corrected accordingly:

$$
S^{*}(n)=S(n) \cdot e^{n / \tau^{*}}
$$

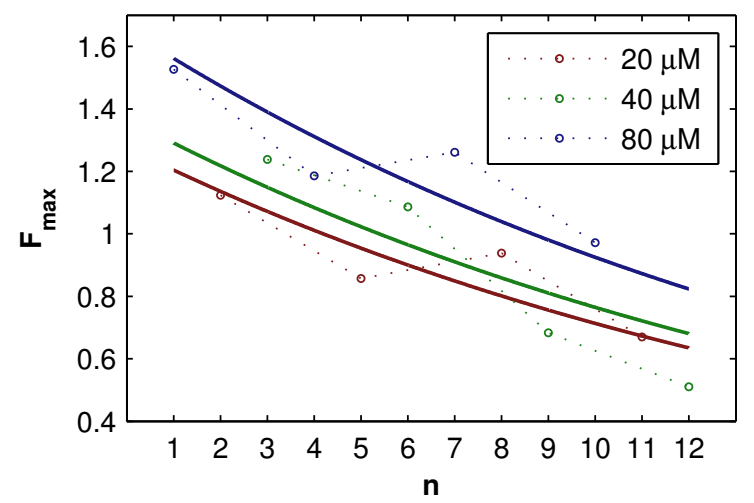

Figure 3.3: Effect of photobleaching on maximum response amplitudes. The maximum response $F_{\max }$ of a single ORN to alternating odor concentrations shows an exponential decay in addition to its concentration dependence (circles, see legend for concentrations). If the amplitudes were to be pooled and averaged over similar concentrations, a systematic error would arise solely based on the order of applications. To compensate for this effect, the set of all amplitudes of one cell were fitted simultaneously by an exponental function (solid lines), including 3 concentration-dependent amplitudes. 


\begin{tabular}{|r|ccc|c|}
\hline & $20 \mu \mathrm{M}$ & $40 \mu \mathrm{M}$ & $80 \mu \mathrm{M}$ & all \\
\hline$t_{0}-t_{\mathrm{m}}$ & & & & \\
mean [ms] & 38.3 & 10.1 & -47.4 & 0 \\
error of the mean [ms] & 26.7 & 25.6 & 31.2 & 16.2 \\
standard deviation [ms] & 358.2 & 341.2 & 421.8 & 376.8 \\
significance & $*$ & & $*$ & \\
\hline$t_{0}-t_{\mathrm{r}}$ & & & & \\
mean [ms] & 38.3 & 10.1 & -47.4 & 0 \\
error of the mean [ms] & 22.9 & 19.3 & 26.7 & 13.5 \\
standard deviation [ms] & 306.9 & 257.4 & 361.1 & 313.4 \\
significance & $*$ & & $*$ & \\
\hline
\end{tabular}

Table 3.1: Comparison of statistics between relative latencies $t_{0}-t_{\mathrm{m}}$ and $t_{0}-t_{\mathrm{r}}$

\subsubsection{Distribution and concentration dependency of re- sponse parameters}

The response parameters $t_{0}, F_{\max }^{*}$ and $S^{*}$, pooled into the three sets of concentrations (20,40 and $80 \mu \mathrm{M})$ for all measurements, are shown in Fig. 3.4. In the following, subsets of parameters are referred to by using the indices $m$ (slice number), $n$ (application number for each slice) and $r$ (cell (ROI) number).

Response onset latencies The absolute time points $t_{0}$ of the onset latencies were not comparable between measurements of different slices, as the position of the application system and therefore the time of stimulus varied from slice to slice. The simplest correction was to use the average of $t_{0}$ for all responses of one slice $\left(=t_{\mathrm{m}}\right)$ and to individually subtract it from the corresponding set of $t_{0}$. The result is plotted as a function of the concentration in Fig. 3.4 A. The relative latencies decrease with increasing concentration in a linear relationship for the observed concentration range, but vary considerably (cf. histograms and Table. 3.1). As a result, the difference is significant (two-sample t-test, $p=0.05$ ) only between the concentrations 20 and $80 \mu \mathrm{M}$. The averaged population latency thus encodes the investigated concentration range only weakly.

Neurons could, however, exhibit different specific onset latencies in ad- 

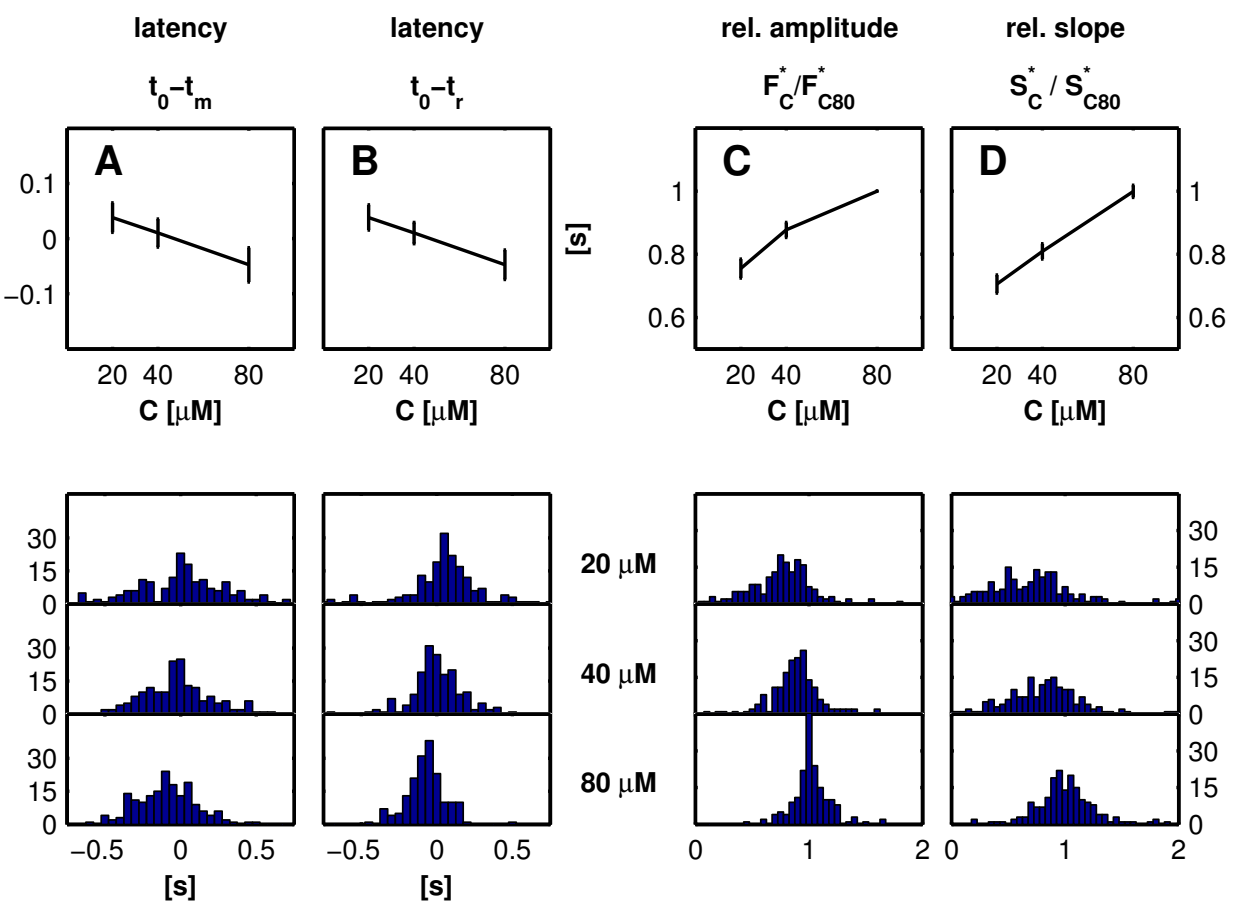

Figure 3.4: Population response statistics for a single odor. Different properties of ORN responses are plotted as a function of odor concentration in the top row, with individual distributions for each concentration shown below. Data is obtained from 540 responses ( $57 \mathrm{ORNs}, 12$ slices). (A) Response onset time $t_{0}$ relative to the average response onset time $t_{\mathrm{m}}$ of all cells and all applications in the measured slice, with error bars as the standard error of the mean. $t_{0}-t_{\mathrm{m}}$ decreases for higher concentrations, but the difference is significant only between 20 and $80 \mu \mathrm{M}$ (two-sample t-test, $p=0.05$ ). From the individual histograms below, it is clear that individual latencies are distributed very broadly. (B) Response onset time $t_{0}$ relative to the average response onset time $t_{\mathrm{r}}$ of all applications for the individual cell. The variation is slightly reduced by removing inter-cell latency differences, but the average onset latency still differs significanty only between 20 and $80 \mu \mathrm{M}$. (C) Photobleaching-corrected fluorescence amplitudes $F_{\mathrm{C}}^{*}$ (for concentrations C) in relation to the fluorescence amplitudes at $80 \mu \mathrm{M}\left(F_{\mathrm{C} 80}^{*}\right)$. The relative amplitudes increase for higher concentrations (all concentration pairs significant), but a saturation at $80 \mu \mathrm{M}$ is already visible. (D) Photobleaching-corrected fluorescence slopes $S_{\mathrm{C}}^{*}$ (for concentrations $\mathrm{C}$ ) in relation to the slopes at $80 \mu \mathrm{M}\left(S_{\mathrm{C} 80}^{*}\right)$. Slopes linearly increase with concentration (all pairs significant). 
dition to their concentration dependence, encoding the type of odor by the vecor of their onset latencies (as observed for mitral cell activity in the olfactory bulb [23]). To test for the contribution of this effect to the distribution of latencies, the individual average $t_{\mathrm{r}}$ of each ORN latency was subtracted from $t_{0}$ (Fig. 3.4 B, Table 3.1). The histograms for $t_{0}-t_{\mathrm{r}}$ show a slightly reduced variance, but it does not improve the significance between concentrations, suggesting that latency coding does not dominate the observed variance.

Fluorescence amplitudes The maximum fluorescence amplitude is often used in $\left[\mathrm{Ca}^{2+}\right]_{i}$ imaging to quantify neuronal responses, and is therefore plotted in Fig. 3.4 C. To individually assess the concentration dependence for each cell in a comparable way, the amplitudes $F_{\max }^{*}$ of concentrations $\mathrm{C}$ $\left(=F_{\mathrm{C}}^{*}\right)$ are divided by the average of the corresponding amplitudes $F_{\mathrm{C} 80}^{*}$ for $80 \mu \mathrm{M}$. This ratio increases with higher concentrations in a significant way (between all pairs of concentrations), but a saturation effect is observable already at $80 \mu \mathrm{M}$.

Fluorescence slopes The fluorescece slopes are shown in Fig. 3.4 D, similarly normalized to the slopes at $80 \mu \mathrm{M}$. The relative slope $\left(S_{\mathrm{C}}^{*} / S_{\mathrm{C} 80}^{*}\right)$ linearly and significantly increases with concentration for the observed range. The histograms show a broader distribution of relative slopes for lower concentrations, potentially indicating different dynamic ranges of individual ORNs: while some ORNs increase their slope to a large degree between stimuli at 20 and $80 \mu \mathrm{M}$, others might be already saturated. If slopes are interpreted as action potential frequencies, these findings support the concept of combinatorial odor coding, in a sense that individual ORN sensitivity is encoded in action potential frequencies.

\subsection{Calcium ion influx in single ORNs}

In the previous section, it was not possible to reliably resolve fast bursts of action potentials (APs) in somata of olfactory receptor neurons by imaging the intracellular calcium ion concentration $\left(\left[\mathrm{Ca}^{2+}\right]_{i}\right)$ via $\left[\mathrm{Ca}^{2+}\right]$ sensitive dyes. 
This might relate to an overlap of different $\left[\mathrm{Ca}^{2+}\right]_{i}$ sources, of which only voltage-gated $\mathrm{Ca}^{2+}$ channels (VGCC) might contribute in an AP-synchronized manner.

To clarify the contribution of VGCCs to the overall $\left[\mathrm{Ca}^{2+}\right]_{i}$ dynamics and their distribution over the cell, an experimental technique fulfilling the following requirements needs to be employed:

1. The cell has to be depolarized without activation of the transduction cascade in order to record $\mathrm{Ca}^{2+}$ influx specifically from VGCCs.

2. $\left[\mathrm{Ca}^{2+}\right]_{i}$ is to be measured with high temporal resolution, preferably in the range of single action potentials.

3. The spatial resolution should be sufficient to distinguish between membrane areas and other possible sources of $\left[\mathrm{Ca}^{2+}\right]_{i}$ (e.g. internal storages).

4. For a higher signal-to-noise ratio, several repetitions of a depolarization pulse need to be averaged or correlated.

5. Each depolarization pulse should generate a sufficient cocentration difference $\Delta\left[\mathrm{Ca}^{2+}\right]_{i}$ to be detected, but $\left[\mathrm{Ca}^{2+}\right]_{i}$ should not accumulate over time.

These conditions can be principally met by a combination of the patchclamp technique and fast $\left[\mathrm{Ca}^{2+}\right]_{i}$ imaging. The time and length of depolarization pulses can be easily controlled in the voltage clamp mode after the cell has been brought into the whole cell configuration. The intracellular solution can be controlled via the pipette solution, including the concentrations of a fast $\left[\mathrm{Ca}^{2+}\right]$ sensitive dye and $\mathrm{Ca}^{2+}$ buffers to prevent $\left[\mathrm{Ca}^{2+}\right]_{i}$ accumulation. Energy can be provided via ATP, GTP and phosphocreatine.

High spatial and temporal resolution, signal-to-noise ratio, and a low degree of photobleaching are, however, conflicting objectives, for which a compromise had to be found in the parameters of the imaging system. 


\subsubsection{Fast confocal imaging in frame mode $(y t z)$}

In the first set of experiments, the confocal line illumination microscope was used in a conventional scanning order, i.e. moving the excitation line over the image area in the $\mathrm{y}$-direction and interpreting the sequence of linear fluorescence distributions as the rows of one image frame at a common point in time. After each time series of frames, the objective was moved to the next z-position (total of 5-7 to cover one cell).

ORNs were patch-clamped in the whole-cell configuration with pipette solutions containing varying concentrations of the $\left[\mathrm{Ca}^{2+}\right]$ sensitive dyes fluo4, fluo-5F, or fluo-5N (100-400 $\mathrm{MM})$ and the $\mathrm{Ca}^{2+}$ chelators EGTA or BAPTA $(0.2,2,5$ and $10 \mathrm{mM})$. The aim was to find optimal conditions in terms of a high relative fluorescence increase that was spatially confined to sites of $\mathrm{Ca}^{2+}$ influx.

For the combination of $100 \mu \mathrm{M}$ fluo-5F and $10 \mathrm{mM}$ EGTA, Fig. 3.5 A depicts the average fluorescence intensity of an ORN that was repeatedly depolarized to $0 \mathrm{mV}$ in pulses of $50 \mathrm{~ms}$ width at a pulse frequency of $2 \mathrm{~Hz}$ and imaged at $45 \mathrm{f} / \mathrm{s}$. The fluorescence time traces $\left(\Delta F / F_{0}\right)$ averaged from selected regions of the cell mostly follow the depolarization pulses, with peaks of higher amplitude in regions of the cell membrane (ROI2, Fig. 3.5 C).

The signals of single pixels, however, were difficult to interpret due to the high level of noise, even when averaged over all time points during depolarization (Fig. 3.5 E). As the evaluation of $\mathrm{Ca}^{2+}$ influx on the spatial scale of single pixels $(350 \mathrm{~nm})$ would be highly desirable, activity correlation imaging [22] was tested as a method to detect pixel traces that are similar to those of manually identified regions of high fluorescence change. Here, the normalized correlation coefficient in the time dimension is calculated between the reference region and each pixel. The resulting correlation maps for the somatic (ROI1) and a membrane (ROI2) signal are shown in Fig. $3.5 \mathrm{~F}$ and G, respectively. While ROI1 correlates with the whole cell, ROI2 correlates more specifically with the cell membrane ${ }^{1}$. The correlation along the membrane

\footnotetext{
${ }^{1}$ Note that correlation maps need to be interpreted with caution in the areas from which the reference trace is obtained. Fig. $3.5 \mathrm{H}$ shows the correlation map for an arbitrary selected test pattern (ROI3) of 10 pixels outside of the cell. Each pixel contributes a
} 
is, however, distributed quite homogeneously, which may have two possible explanations: (i) the distribution of $\left[\mathrm{Ca}^{2+}\right]_{i}$ sources over the membrane is homogeneous on the measured scale, (ii) the limitation of spatial $\left[\mathrm{Ca}^{2+}\right]_{i}$ spread by $\mathrm{Ca}^{2+}$ buffers is not sufficient to resolve sources of $\left[\mathrm{Ca}^{2+}\right]_{i}$ during an image frame of $22 \mathrm{~ms}$.

To improve the spatial resolution in case of (ii), the slow $\mathrm{Ca}^{2+}$ chelator EGTA was substituted with the faster BAPTA (binding rates $k_{o n}^{\text {EGTA }}=$ $\left.2.6 \times 10^{6} \mathrm{M}^{-1} \mathrm{~s}^{-1}, k_{\text {on }}^{\text {BAPTA }}=4 \times 10^{8} \mathrm{M}^{-1} \mathrm{~s}^{-1},[65,73]\right)$. Under these conditions, transient fluorescence signals were not detectable ( $\mathrm{n}>4$ cells), supposedly because BAPTA is sufficiently fast to directly compete with fluo-5F $\left(k_{\text {on }}^{\text {fluo }-5 \mathrm{~F}}=2.36 \times 10^{8} \mathrm{M}^{-1} \mathrm{~s}^{-1}[75]\right)$ at influx sites and thereby reduces the concentration of $\mathrm{Ca}^{2+}$-bound dye.

A second approach was, instead of relying on $\mathrm{Ca}^{2+}$ buffers to create steady-state $\left[\mathrm{Ca}^{2+}\right]_{i}$ profiles, to increase the acquisition speed and decrease the length of depolarization so that $\left[\mathrm{Ca}^{2+}\right]_{i}$ could be observed dynamically. For $110 \mathrm{f} / \mathrm{s}(\mathrm{n}=6)$ and $220 \mathrm{f} / \mathrm{s}(\mathrm{n}=1)$, the correlation maps (created as in $3.5 \mathrm{G})$ still revealed a homogeneous distribution along the membrane similar to the slower measurements. As the reduction of the camera integration time for higher frame rates made it necessary to increase the excitation laser power at least linearly, photobleaching became the limiting factor, and it was impossible to image more than one optical plane under these conditions. For frame rates significantly higher than $100 \mathrm{f} / \mathrm{s}$, a different imaging approach was needed.

\subsubsection{Sequential line imaging (tyz)}

To increase the frame rate without the need for high excitation laser power, the scanning protocol for the LIM was changed in the following way: for a constant position (y) of the illumination line, a time series of 250 linear fluorescence distributions (in $\mathrm{x}$ ) was recorded. The measurement was repeated

random (noise) component to the reference signal (Fig. 3.5 D), and is thereby automatically correlated to ROI3 due to autocorrelation, recreating the original test pattern in the correlation map. Therefore ACI makes only sense if the initially selected reference regions contain signals distiguishable from the background noise. 

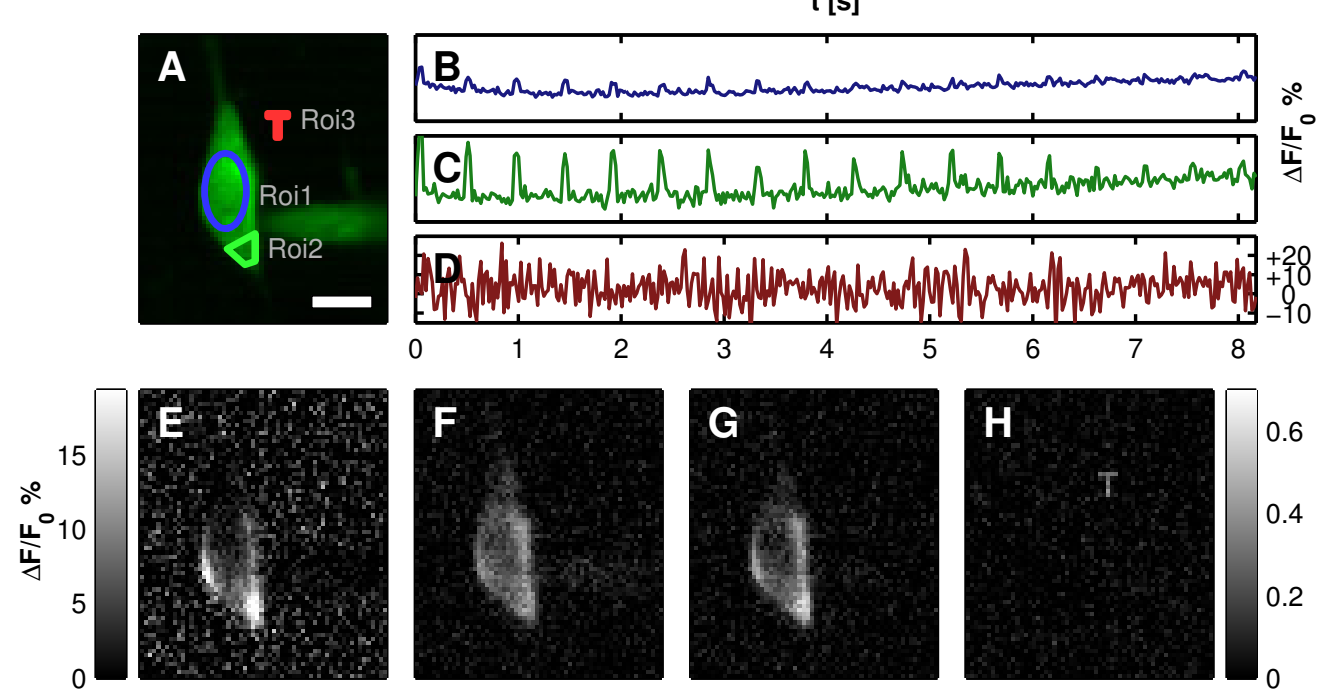

Figure 3.5: $\left[\mathrm{Ca}^{2+}\right]_{i}$ imaging of single ORN with simultaneous depolarization in the whole-cell voltage clamp mode. ORNs were filled with $100 \mu \mathrm{M}$ fluo-5F and $10 \mathrm{mM}$ EGTA via patch clamp in the whole-cell configuration and depolarized in pulses of $50 \mathrm{~ms}$ from $-75 \mathrm{mV}$ to $0 \mathrm{mV}$ in the voltage clamp mode. The acquisition rate is $45 \mathrm{f} / \mathrm{s}$. (A) Time-averaged fluorescence intensity, with three selected regions of interest (ROIs), covering most of the cell soma (ROI1, blue), a membrane area rear the axon (ROI2, green), and an arbitrary test pattern outside of the cell (ROI3, red). Scale bar $5 \mu \mathrm{m}$. (B-D) Corresponding ROI time traces $\left(\Delta F / F_{0}\right)$ show larger transient fluorescence increase in the membrane area than in the cell soma. (E) Fluorescence increase $\left(F_{s}-F_{0}\right) / F_{0}$ calculated from averaged fluorescence intensities during $\left(F_{s}\right)$ and between stimulations $\left(F_{0}\right)$. ( $\left.\mathbf{F}-\mathbf{H}\right)$ ACI maps using reference traces from ROI 1-3, respectively. The somatic signal exhibits moderate correlation to the whole cell area $(\mathrm{F})$, while the signal from ROI2 correlates highly and specifically with the majority of the membrane area $(\mathrm{G})$ of the cell. $(\mathbf{H})$ Interestingly, ROI3 highly correlates with all pixels that contribute to ROI3, demonstrating a limitation of ACI in case of low signal-to-noise ratio. 
at $1 \mathrm{~Hz}$ for $32 \mathrm{y}$-positions, and repeated again for 5-7 z-positions to sample most of the cell in 3 dimensions and time.

For the sequential line measurements to be interpreted as samples of the same prototypic four-dimensional $\left[\mathrm{Ca}^{2+}\right]_{i}$ distribution, the timing of the depolarization pulses during each measurement had to be well-synchronized to the acquisition times of the CCD detector. However, the triggering of fast depolarization pulses via the software of the patch-clamp equipment was not possible in the required precision. To solve this problem, a custom electrical circuit was designed and built using an analog switch that could rapidly $(<250 \mathrm{~ns})$ change the command voltage of the patch-clamp amplifier to a user-defined potential. The trigger pulses were generated by the software for the scanning mirror system together with the trigger pulses for the integration times of the CCD detector, guaranteeing synchrony between mirror position, CCD acquisition times and depolarization pulses.

Using this scanning mode, the effective frame rate could be increased to $1250 \mathrm{f} / \mathrm{s}$, while the excitation intensity could be lowered by at least a factor of 4 (due to increased pixel dwell times), thereby reducing photobleaching.

A typical measurement of a ORN is shown in Fig. 3.6 for a line position that intersects a cell region of elevated $\mathrm{Ca}^{2+}$ influx. During the measurement time of $200 \mathrm{~ms}$, the cell was depolarized in three pulses (to $-10 \mathrm{mV}$ ) of $6.4 \mathrm{~ms}$ width (Fig. 3.6 A). In the spatiotemporal fluorescence distribution along the line (Fig. 3.6 B, time on the horizontal axis), a highly localized fluorescence increase is clearly visible. It is synchronized to the depolarization pulses, but does not follow it in a linear relationship. In the plot of the fluorescence intensity for the small region of $\mathrm{Ca}^{2+}$ influx (Fig. 3.6 D), there is a steep increase during the depolarization pulses that is far from reaching saturation, which proves that indeed a dynamic $\left[\mathrm{Ca}^{2+}\right]_{i}$ signal is measured instead of a steady-state profile.

\subsubsection{Analysis of $4 \mathrm{D}$ data using model-driven ACI}

As in the previous section, the averaged ROI signal (Fig. 3.6 D) could be used directly for ACI to detect pixels similar to the influx ROI. However, a 


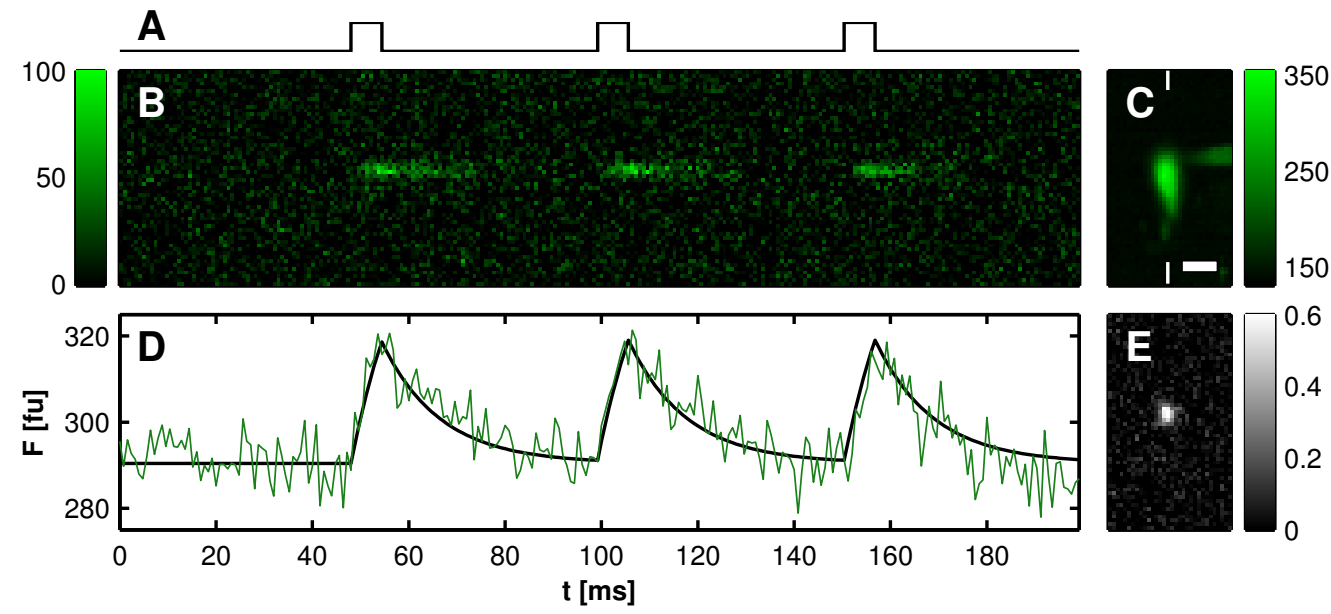

Figure 3.6: Fast $\left[\mathrm{Ca}^{2+}\right]_{i}$ imaging using sequences of line scans acquired at 1250 $\mathrm{f} / \mathrm{s}$. (A) For each line scan, the cell was depolarized to $-10 \mathrm{mV}$ using three pulses of $6.4 \mathrm{~ms}$ duration. (B) Corresponding spatiotemporal fluorescence evolution $\Delta F=$ $F-F_{0}$ for one line scan through the cell soma, color-coded according the color map on the left. (C) Initial fluorescence $F_{0}$ for all 32 line scans that cover one optical plane of the cell, color-coded according to the color map on the right. Vertical markers indicate the line position corresponding to B. Scale bar $5 \mu \mathrm{m}$. (D) Raw fluorescence data from the region of high $\Delta F$ (green line), and least-squares bestfit result (black line) using a simplified numerical model of $\left[\mathrm{Ca}^{2+}\right]_{i}$ dynamics near the membrane. (E) The correlation map between the best-fit curve from D and individual pixel traces from the line scans shows a highly correlated area. The contribution of photon shot noise autocorrelation (as in Fig. $3.5 \mathrm{H}$ ) is already eliminated by using the best-fit curve as reference trace instead of the original fluorescence data. 
major disadvantage of this approach are other sources of correlation like pixel autocorrelation (see Fig. 3.5 H), laser intensity fluctuations, and electrical CCD detector oscillations, which contaminate the resulting correlation map and make it less meaningful.

Can this situation be improved? Ideally, one would use a reference signal that is cleaned from any noise or other effects irrelevant to the physical system of interest. This can in fact be achieved by fitting a numerical model to the ROI signal which only reflects $\left[\mathrm{Ca}^{2+}\right]_{i}$ dynamics from known experimental conditions. The curve of the fit result can then be used for ACI instead of the original data. A very simple model that follows the observed fluorescence intensity is:

$$
\begin{gathered}
I_{n}=c_{\text {decay }} I_{n-1}+a_{\text {in }} S_{n} \\
F_{n}=I_{n}+F_{0}
\end{gathered}
$$

with the fluorescence count $F_{n}$ (time $\mathrm{t}$ is discretized as $t=n \Delta t$ with samples times $\Delta t$ and sample index $n$ ), the resting fluorescence $F_{0}$, a parameter $c_{\text {decay }}<1$ summarizing all effects of $\left[\mathrm{Ca}^{2+}\right]_{i}$ reduction $\left(\mathrm{Ca}^{2+}\right.$ buffering, extrusion, diffusion), the influx amplitude $a_{\text {in }}$ and the depolarization state $S_{n}$ (=1 during depolarization, 0 otherwise). After a least-squares fit to the ROI signal using the two parameters $a_{\text {in }}$ and $c_{\text {decay }}$, the curve describes the fluorescence trace quite well (Fig. 3.6 D, plotted as black line).

The corresponding three-dimensional correlation map using the fit result as reference trace is depicted in Fig. 3.7 A-G. It is reproducable with data from a second measurement on the same cell and shows several hotspots heterogeneously scattered over the cell membrane. In order to distinguish between the background and pixels of significantly elevated correlation, it is useful to consider the histogram of all correlation coefficients in the map (Fig. $3.8 \mathrm{~A}, \mathrm{C}$ ). The majority of correlation values are distributed in a gaussian shape, with only a small fraction deviating towards positive correlations (best seen in the logarithmic scale). The gaussian shape of the histogram is to be expected from uncorrelated pixel traces of sample length $\mathrm{N}$, with mean $\mu=0$ and $\sigma=\frac{1}{\sqrt{N-3}}[13]$. This value can be verified by fitting a histogram of a correlation map generated from an uncorrelated image area (Fig. 3.8 B,D, 
$\left.\sigma_{\text {fit }}=0.0632, \sigma_{\text {theory }}=0.0637\right)$. By calculating a threshold correlation $c_{t h}$ at the intersection of the gaussian curve with a histogram value of 1 , all significantly correlated pixels can be selected, with only one false positive per correlation map.
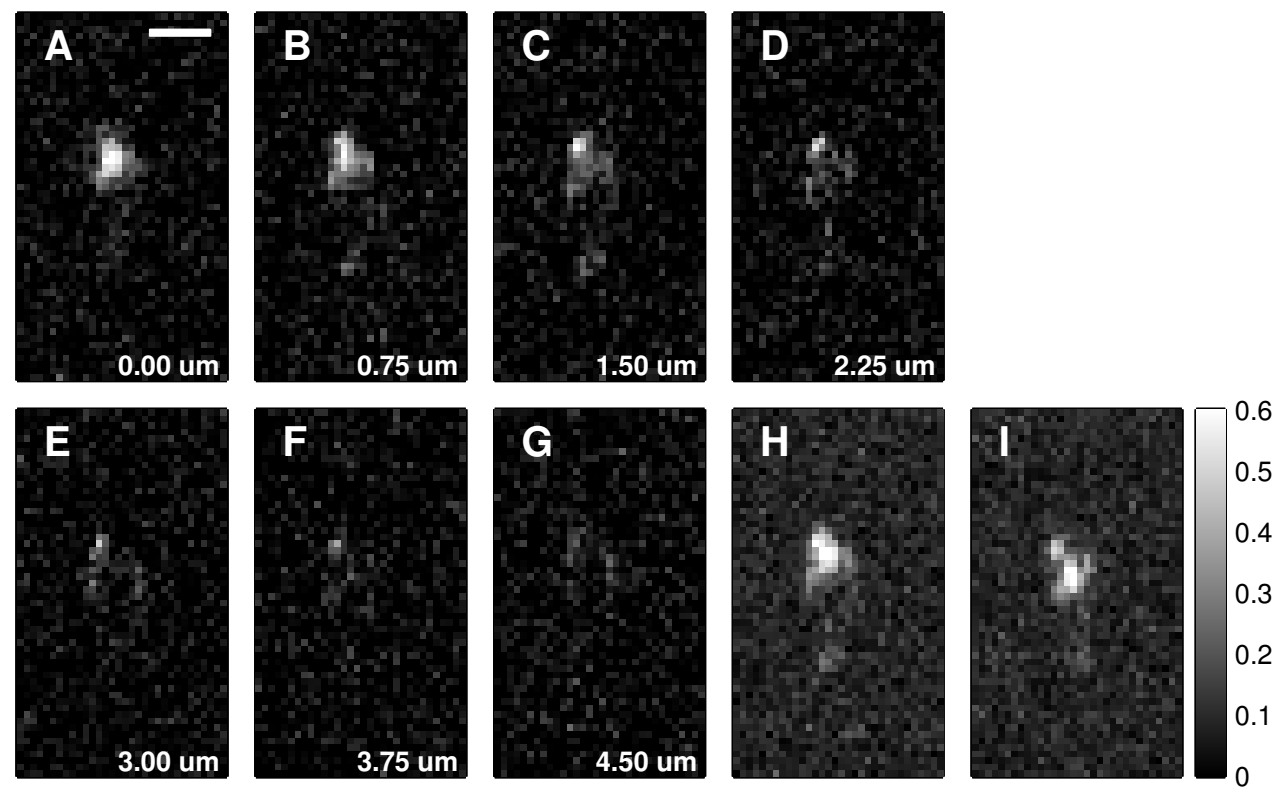

Figure 3.7: Three-dimensional correlation map and comparison with a repetiton of the experiment. (A-G) The correlation map as in Fig. $3.6 \mathrm{E}$ is shown for 7 different optical layers at axial intervals of $0.75 \mu \mathrm{m}$. A highly heterogeneous distribution of the correlation coefficient is visible, with most regions of high correlation located at the membrane near the axon. Scale bar 5 um. (H) Maximum projection over all planes. (I) Similar maximum projection from a repetition of the experiment after $15 \mathrm{~min}$. Slight differences might result from a deformation of the cell due to drift of the position of the patch pipette over time.

\subsubsection{Pixel-wise fit of time traces}

As the last step of the image analysis, all pixel traces that were significantly correlated to the modelled $\mathrm{Ca}^{2+}$ influx (and therefore contained meaningful temporal information) were individually fit again by the same numerical model, yielding fit values for $\mathrm{Ca}^{2+}$ influx amplitude $a_{\text {in }}$ and decay constant 

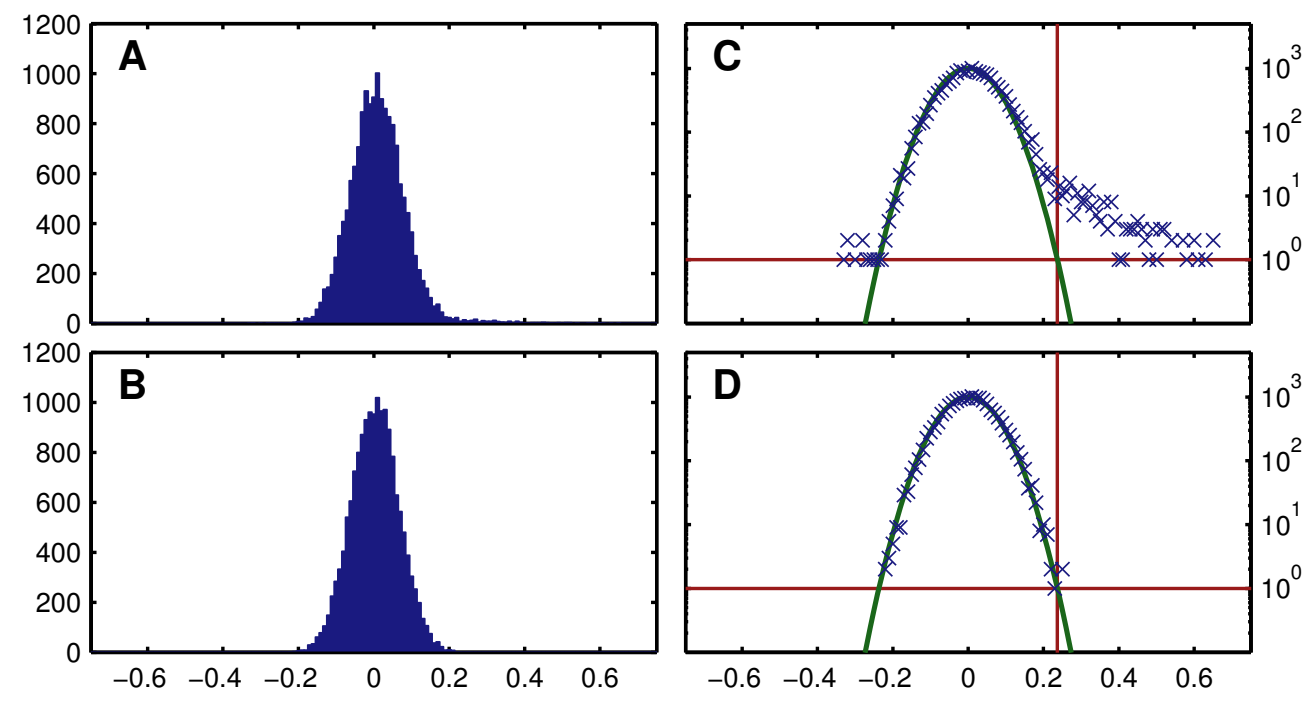

Figure 3.8: Histograms of correlation maps. (A) The correlation coefficients of the 3D correlation map shown in Fig. $3.7 \mathrm{H}$ are approximately distributed in a gaussian shape with mean $\mu=0$ and several outliers in the positive direction. (B) Similar plot for a correlation map created from a different region (located at $35 \mu \mathrm{m}$ from the cell area, data acquired at the same time), with no outliers due to missing signal correlation. (C) Histogram from A in half-logarithmic scale, clearly showing the contribution of signal correlation as a deviation from the gaussian shape (quadratic in logarithmic plot). (D) Half-logarithmic plot of histogram B, with best-fit (green line) using a gaussian function (mean $\mu=0$, fit parameters $A$ (amplitude) and $\sigma$ (width)). The intersection of the fit curve with a histogram value of 1 (red horizontal line) is chosen as threshold correlation value (red vertical line) to separate significant signal correlations from the main distribution, with an average of one false-positive per correlation map. 
$c_{\text {decay }}$ for each pixel. These values can be seen as indicators of the distance of the pixel position to a $\left[\mathrm{Ca}^{2+}\right]_{I}$ source, with higher $a_{\text {in }}$ and lower exponential time constants $\tau_{\mathrm{d}}=-\Delta t \log \left(c_{\text {decay }}\right)$ signifying a lower distance to the source (for further elaboration of $\left[\mathrm{Ca}^{2+}\right]_{i}$ dynamics near a membrane, see [43] (steady-state) and [25] (partly dynamic)). The fit parameters for all pixel traces pooled over 8 different experiments are shown in Fig. 3.9. For comparability between different cell conditions, the amplitudes $a_{\text {in }}$ were normalized with the inital ROI fit parameter $a_{\mathrm{ROI}}$ of the corresponding cell before pooling. From the distribution of $a_{\mathrm{in}} / a_{\mathrm{ROI}}$ it is evident that the majority of pixel traces represent lower influx amplitudes than the manually selected ROI, but are still detected by the correlation method. The fluorescence decay times have an average value of $13.4 \mathrm{~ms}$ and are distributed over a broad range of 5-25 ms. The decay time also slightly decreases as a function of amplitude (Fig. $3.9 \mathrm{C}$ ), in accordance to the previous interpretation. The anticorrelation between $a_{\mathrm{in}} / a_{\mathrm{ROI}}$ and $\tau_{\mathrm{d}}$ is, however, not very strong $\left(c_{\mathrm{cor}}=-0.24\right)$ suggesting that the two parameters might contain independent information.
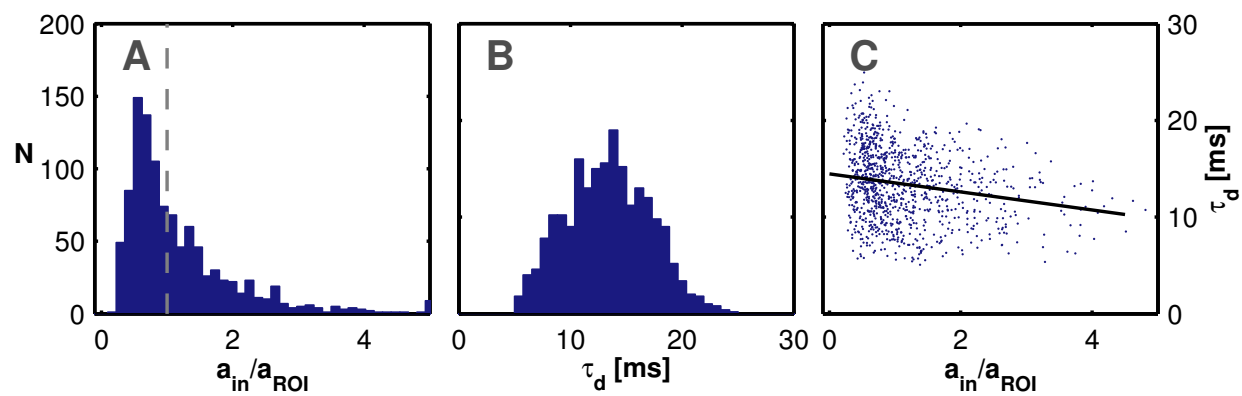

Figure 3.9: Fit Parameters for individual pixels, pooled over all experiments. Pixels were selected by cross-correlation thresholds, and their fluorescence traces were individually fitted by a theoretical model as in Fig. 3.6 D. (A) Distribution of normalized $\mathrm{Ca}^{2+}$ influx amplitudes $\left(a_{\text {in }} / a_{\mathrm{ROI}}\right)$ during depolarization. Large positive values are expected to represent regions in vicinity of $\mathrm{Ca}^{2+}$ channels. Dashed line: average ROI amplitude of 1 . (B) Distribution of fluorescence decay time $\tau_{d}$. Fast kinetics are expected near $\mathrm{Ca}^{2+}$ channels. (C) Relationship between amplitudes and time constants.

Both parameters should therefore be used in a spatial map describing the 
sources of $\left[\mathrm{Ca}^{2+}\right]_{i}$. To this end, the ratio $a_{\text {in }} / \tau_{\mathrm{d}}$ was chosen, as the intercell normalization with $a_{\mathrm{ROI}}$ is unnecessary for single cells. The ratios are displayed in the green channel for 8 measurements in Fig. 3.10 as maximum z-projections of the three-dimensional ratio maps. For comparison, the initial fluorescence $F_{0}$ is shown in the red channel.

\subsubsection{Distribution of VGCCs in ORN somata and total calcium ion current}

As a general observation, regions of $\mathrm{Ca}^{2+}$ influx are inhomogeneously distributed over the cell somata and usually organized into several clusters. These clusters may have the role to facilitate functional $\mathrm{Ca}^{2+}$ microdomains in combination with large conductance $\left[\mathrm{Ca}^{2+}\right]_{i}$-dependent $\mathrm{K}^{+}$channels, which is discussed in the next section.

The whole-cell $\mathrm{Ca}^{2+}$ current contributed by VGCCs is roughly estimated by the steady-state current during depolarization pulses, after the transient inward $\mathrm{Na}^{+}$current has subsided due to inactivation. $\mathrm{K}^{+}$currents were blocked by Tetraethylammonium (TEA) in the pipette solution (in which also $\mathrm{K}^{+}$is replaced by $\mathrm{Cs}^{+}$). As an upper limit (as $\mathrm{Na}^{+}$inactivation was not complete after $6.4 \mathrm{~ms}$ ), steady-state currents from the experiments shown in Fig. 3.10 were $80 \mathrm{pA}$.

As a comparison, the maximum $\mathrm{Ca}^{2+}$ current through CNG channels of a single cilium in Rana pipiens isolated ORNs is about 300 pA [24]. Assuming a low number of 3 cilia per cell, the maximum whole-cell CNG current would already be one order of magnitude larger than the current through VGCCs. With typical AP durations comparable or less than the depolarisation length of $6.4 \mathrm{~ms}$, the relative VGCC contribution to $\left[\mathrm{Ca}^{2+}\right]_{i}$ is even lower. The diffusion length of $\mathrm{Ca}^{2+}(x=2 \sqrt{D t})$ is $50 \mu \mathrm{m}$ after $1 \mathrm{~s}$, already heavily contanminating $\left[\mathrm{Ca}^{2+}\right]_{i}$ from VGCCs if not removed by $\mathrm{Ca}^{2+}$ extrusion on its way through the dendrite. The $\left[\mathrm{Ca}^{2+}\right]_{i}$ can therefore only be purely related to VGCCs if it is measured shortly after the onset of the response, a condition which is used in the previous section to estimate relative AP frequencies. 

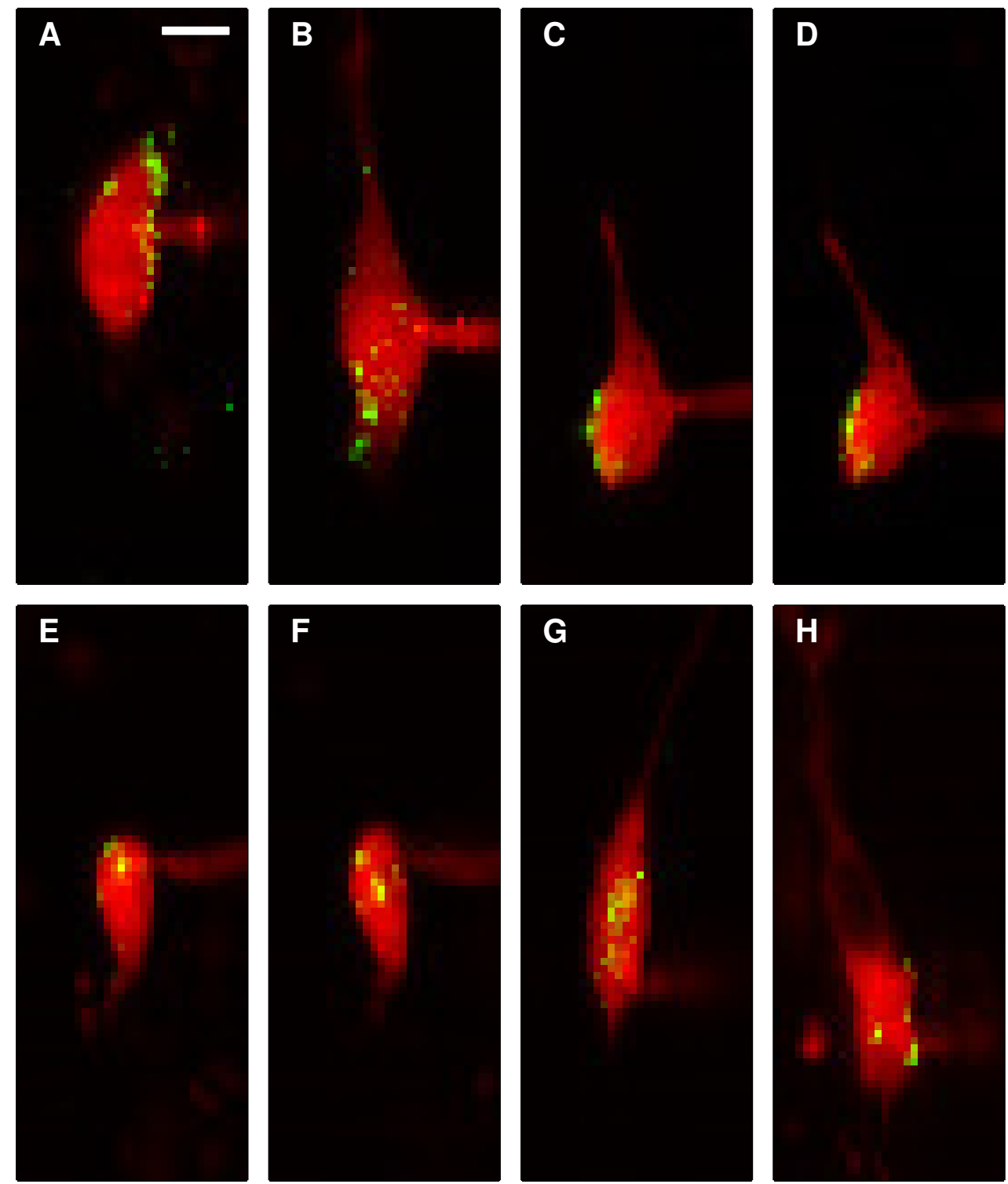

Figure 3.10: Maps of fit parameters showing regions of high $\mathrm{Ca}^{2+}$ influx. (A$\mathbf{H})$ The per-pixel ratio $\frac{a_{i n}}{\tau_{\mathrm{d}}}$ between influx amplitude and decay time constant is displayed in the green channel for each experiment, maximum-projected in the $\mathrm{z}$ direction. The mean fluorescence intensity $F_{0}$ is shown in the red channel. Maps have been scaled to a range of $[0,1]$ for comparability between channels. In the case of two cells ( $\mathrm{C}$ and $\mathrm{E})$, a second measurement after 10-15 minutes was possible (D and $\mathrm{F}$, respectively), showing very similar distributions of $\mathrm{Ca}^{2+}$ influx sites. Scale bar $5 \mu \mathrm{m}$, applicable to all images. 


\subsection{Effect of BK channel blocker iberiotoxin}

The discovery of localized $\mathrm{Ca}^{2+}$ influx in single ORNs immediately lead to the question of its possible function. As one component of Xenopus laevis ORNs currents is known to be a $\left[\mathrm{Ca}^{2+}\right]_{i}$ dependent $\mathrm{K}^{+}$current [59], it is well possible that the corresponding $\mathrm{K}^{+}$channels are located in proximity to the $\left[\mathrm{Ca}^{2+}\right]_{i}$ sources to be activated quickly. During further investigations in our lab (see Discussion), a large conductance $\mathrm{K}^{+}$channel (also called BK channel) has indeed been found to colocalize with the general areas of increased $\mathrm{Ca}^{2+}$ influx. One open question was to what degree ORN population responses would be altered when its function was blocked by iberiotoxin.

To this end, ORN responses to a broad olfactory stimulus (15 amino acids) were measured similar to conditions described in 3.2. First, 2-3 initial responses were recorded. Then, the perfusion system was stopped and the slice preparation incubated for 3-5 min with iberiotoxin (200 nM in Ringer solution, $k_{D}=1.16 \mathrm{nM}$ for its binding to BK channels [5]) for one group of experiments (10 slices, 55 cells), and with only Ringer solution for a control group (4 slices, 22 cells). After the incubation, the perfusion was started again and responses to amino acids were recorded every $3 \mathrm{~min}$ as often as possible.

Fig. 3.11 A and B shows the evolution of response amplitudes $F$ for the control group and the iberiotoxin group, respectively, normalized to the average response amplitude $F_{\text {pre }}$ of measurements before the incubation ("pre") for each cell. The experiment number for each slice is denoted with $n$, with values $<0$ before incubation. As there was a general decrease in $F / F_{\text {pre }}$ in addition to possible effects from iberiotoxin, the contribution of photobleaching could not be reliably modeled and therefore not corrected. Instead, the relative amplitudes are plotted in logarithmic scale, with expected straight lines for a exponential decay dominated by photobleaching (as can be seen in the control group). For a subpopulation in the iberiotoxin group, amplitudes decrease more than exponentially after the incubation time. A quantification of this effect is shown in Fig. $3.11 \mathrm{C}$, where all amplitudes are pooled for both groups into three bins of experiment numbers. The most significant dif- 
ference between iberiotoxin and the control group exists for experiments 3-9 min after incubation, with a less significant difference thereafter, indicating a partial washout effect.
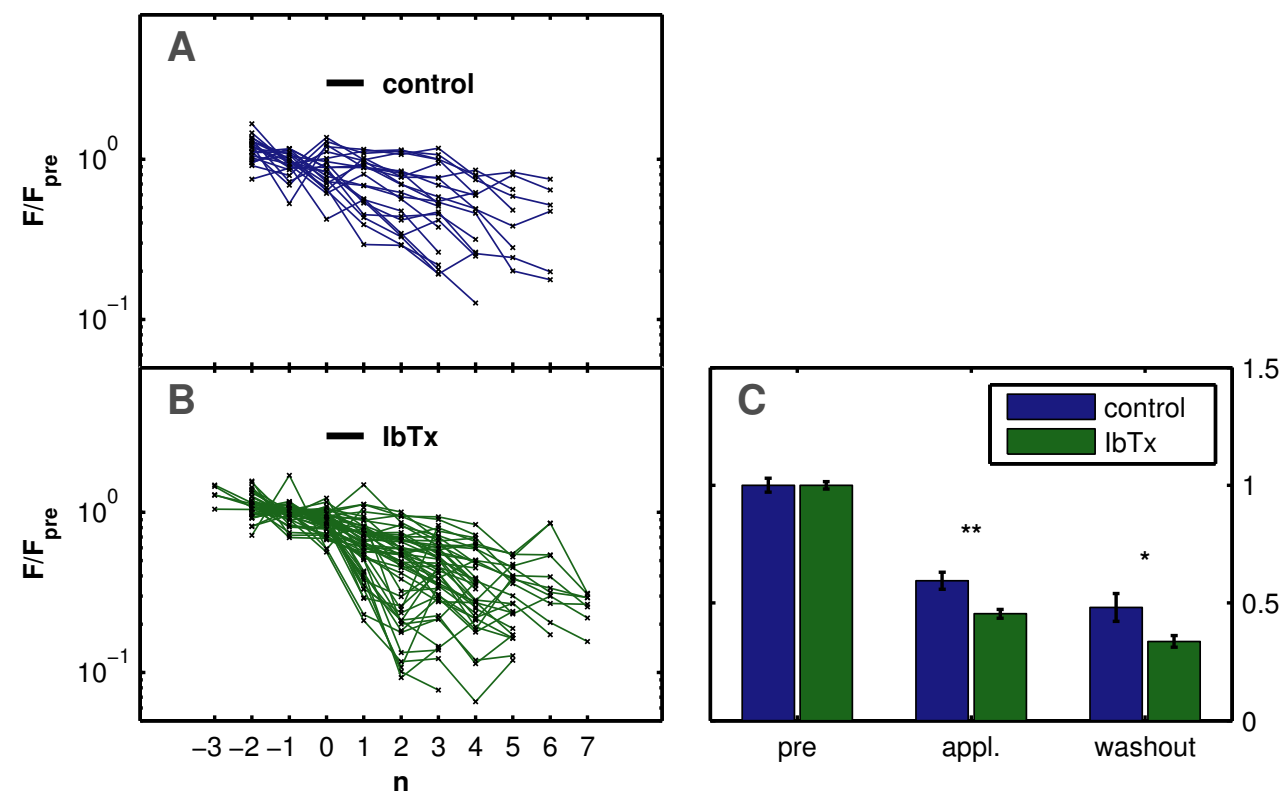

Figure 3.11: Partial reduction of response amplitudes after incubation with iberiotoxin. (A-B) Fluorescence amplitudes of ORN responses to amino acid mixture, plotted over application number $n$. For comparison, each amplitude $F$ is normalized to the averaged amplitudes $F_{\text {pre }}$ before incubation $(n<1)$ for each cell. Slices were incubated between $n=0$ and $n=1$ for 3-5 min with Ringer solution (A) as control or iberiotoxin (200 nM in Ringer solution, B). In addition to an exponential decrease due to photobleaching (straight lines in logarithmic scale), some ORNs exhibit a further reduction after iberiotoxin incubation. (C) Amplitudes are pooled over sets n ("pre": $n<1$, "appl.": $2 \leq n \leq 4$, "washout": $n>4$ ) and compared between control and iberiotoxin. There is a significant (two-sample t-test, $p=0.001$ ) decrease after iberiotoxin incubation, which becomes less significant $(p=0.05)$ after 10-15 min washout. A complete recovery comparable to the control conditions is, however, not observed. 


\subsection{X-Ray phase contrast microscopy}

Different preparation and staining techniques were evaluated for Xenopus laevis tadpoles to be imaged with X-Ray microscopy.

In the first set of preparations, unstained samples were compared in terms of X-Ray image contrast. Here, unfixed samples in water immersion provided the poorest image quality due to high absorption in water and the low difference in diffractive index between tissue and medium. The best conditions for phase reconstruction were achived for tadpoles fixed in 1,5\% glutaraldehyde and $1,5 \%$ paraformaldehyde and supercritically dryed in graded ethanol. An example of a X-Ray micrograph for a fixed sample is given in Fig. 3.12, where fine structures at tissue borders are well visible. A tomographic reconstruction from a rotation series of images is shown in Fig. 3.13 and demonstrates the capabilities of X-Ray phase contrast tomography.

To investigate large-scale three-dimensional correlations between ORN and glomerular or mitral cell populations, it would be necessary to specifically stain neurons in a way detectable by X-Ray microscopy. To this end, Nanogold was chosen as contrast agent $([7,19])$ in a second set of preparations.

Tadpoles were exposed to high odorant concentrations in combination with FluoroNanogold in order to specifically stain ORN populations due to ligand-induced endocytosis $[9,10]$. As a control, electroporation was used to introduce FluoroNanogold unspecifically into ORNs. However, no additional contrast was detectable in the X-Ray micrographs for both staining methods, suggesting that Nanogold might be principally unable to generate image contrast for X-Ray phase contrast microscopy, at least at the attainable concentrations. 


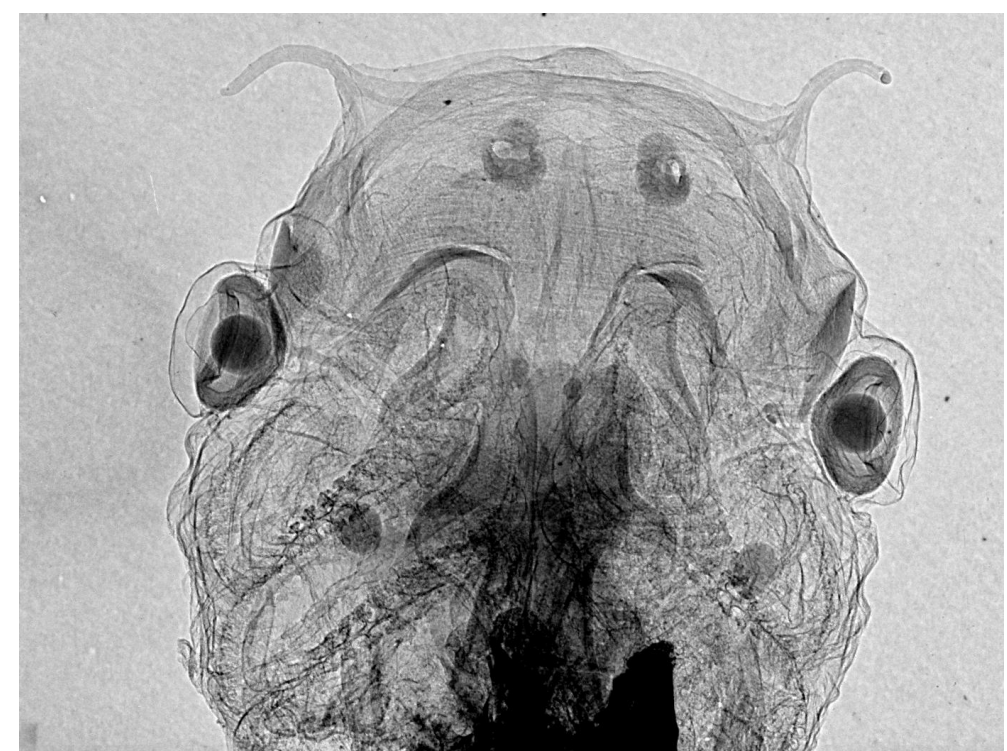

Figure 3.12: X-Ray micrograph of fixed Xenopus laevis tadpole after background correction. Image kindly provided by Martin Krenkel, Institute of X-Ray Physics, University of Göttingen.

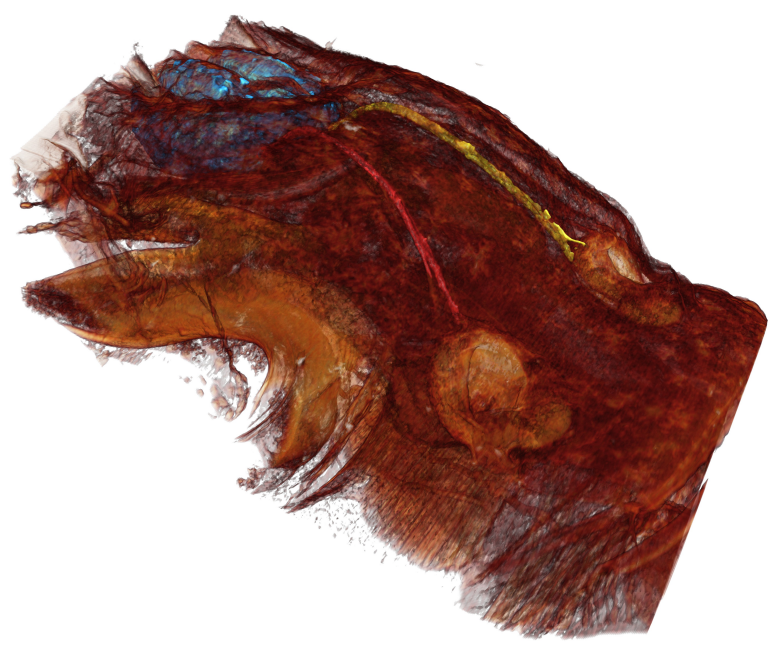

Figure 3.13: Rendering of phase-reconstruced X-Ray tomography, reconstructed from 1830 pictures taken from different angles (increment $0.1^{\circ}$ ). Olfactory nerves (red, yellow) and forebrain (blue) marked by segmentation. Image kindly provided by Martin Krenkel, Institute of X-Ray Physics, University of Göttingen. 


\section{Chapter 4}

\section{Discussion}

\subsection{Conclusions}

Temporally resolved olfactory coding on the level of olfactory receptor neuron (ORN) populations is poorly understood. Motivated by recent findings of patterns of response latencies in mitral/tufted $(\mathrm{M} / \mathrm{T})$ cells in the olfactory bulb [23], the present study investigated ORN population responses with high temporal resolution and their possible role in the generation of the observed latency patterns. Additionally, fast $\left[\mathrm{Ca}^{2+}\right]_{i}$ dynamics on the small scale of single ORNs was performed to better understand the fluorescence data. Furthermore, X-Ray microscopy was evaluated for its suitability to image large-scale morphology. The main conclusions of these experiments can be summerized as follows:

1. The reliable detection of single action potentials (APs) was not possible on a population level. While a low percentage of ORNs exhibited fluorescence steps attributable to APs, the fluorescence signals of most ORNs increased smoothly even in high temporal resolution. The main reason was found to be a low contribution of voltage-gated $\mathrm{Ca}^{2+}$ channels compared to other sources of somatic $\left[\mathrm{Ca}^{2+}\right]_{i}$.

2. Response onset latencies were only weakly correlated to stimulus concentrations and showed a large variance. Inter-ORN latency patterns 
did not contribute to the variance in a high degree, indicating that latency patterns may not play an important role in olfactory coding on the investigated range of odor concentration $(20-80 \mu \mathrm{M})$.

3. Fluorescence slopes at response onset times were the best predictors of odor concentration. In contrast to the commonly evaluated maximum fluorescence response, the ensemble mean of relative slopes increased linearly with odor concentration. Also, when considered at individual ORN level, slopes increased at different rates with concentration, showing individual ORN sensitivities. Together, these findings suggest that olfactory information is mostly contained in ORN AP frequencies, as they are closely related to fluorescence slopes.

4. Voltage-gated $\mathrm{Ca}^{2+}$ channels were found to be distributed heterogeneously over ORN somatic membranes and mostly form local clusters, facilitating local $\left[\mathrm{Ca}^{2+}\right]_{i}$ microdomains.

5. When the $\left[\mathrm{Ca}^{2+}\right]_{i}$ dependent BK channel was blocked by iberiotoxin, ORN response amplitudes were reduced for a subset of cells.

6. A preparation well-suited for X-Ray phase contrast microscopy was found. However, Nanogold did not introduce a detectable contrast in ORN populations.

The implications of these results and their relation to other findings in the olfactory system is discussed in the following.

\subsection{ORN population measurements}

Previous measurements of ORN odorant responses could be generally divided into three categories. In the first, researchers concentrated on the spatial patterns of ORN activation throughout the olfactory epithelia of different species, using $\left[\mathrm{Ca}^{2+}\right]_{i}$ imaging at low temporal resolutions between 0.5 and 2 $\mathrm{Hz}[31,39,47,70]$. From these measurements, individual ORN sensitivities 
to different odors could be elucidated, and ORNs categorized into classes of similar response patterns.

At the other end of the spectrum, fast response properties such as spike frequency and first-spike latency of single ORNs were investigated with electrophysiological techniques, determining their individual dependence on olfactory stimuli $[16,50,51,55,56]$. From the statistics of these experiments, ORN ensemble responses were usually inferred.

In the recent years, a third category of ORN measurements was established, in which the output to the olfactory bulb was determined by $\left[\mathrm{Ca}^{2+}\right]_{i}$ imaging at the glomerular ORN axon terminals [6, 66, 72]. Here, the average responses of whole ORN classes projecting to the same glomeruli was investigated, and the temporal resolution could be increased because of signal summation over the glomeruli. However, by averaging over ORN classes, information about the individual properties of its members was lost.

The ORN population measurements in this study belong to a fourth category. While not being able to compete with patch-clamp recordings in detecting APs, it was possible to image ORNs responses simultaneously and sufficiently fast to gather temporal response properties on population level, namely the relative AP frequency and the onset latency.

\subsubsection{Onset latencies}

The observed ORN onset latencies (ensemble mean) changed with odor concentrations, being reduced by $85 \mathrm{~ms}$ when the odorant concentration was increased from 20 to $80 \mu \mathrm{M}$. Their variation was high for constant concentrations (Table 3.1, standard deviation up to $422 \mathrm{~ms}$ ), even when corrected for cell-specific average latency (up to $362 \mathrm{~ms}$ ). The concentration dependence of the latency was weak when compared to typical single-cell ORN response latencies from Rana ridibunda [55], where median latencies changed from $2.51 \mathrm{~s}$ to $0.87 \mathrm{~s}$ when concentration was increased by 1.55 (the median dynamic range of concentrations). However, those latency differences were observed at much lower concentrations $(1 \mathrm{nM}-1 \mu \mathrm{M})$. The discrepancy is thus easily explained by ORN latency responses being mostly saturated for the 
measured concentration range. This also means that latency coding (in the sense of odor-specific latency vectors for sets of ORNs) cannot be excluded to exist for low odor concentrations.

\subsubsection{Action potential frequencies}

The relative AP frequency for individual ORNs was determined from the ratio of the fluorescent slope to the slope at maximum concentration. Its ensemble mean increased by a factor of $1.36 \pm 0.03$ between odorant concentrations of 20 and $80 \mu \mathrm{M}$. Individual ORN frequencies increased in a broad range (best seen in Fig. 3.4D, histogram for $20 \mu \mathrm{M}$ ), indicating different dynamic ranges for the response frequency for each ORN. This is in agreement to single-cell statistics, where the range of molarities over which the response frequency increases from 0 to $95 \%$ varies from 0.4 to 4.5 decades depending on neuron and odorant [55]. The concentration is thus well-described by the ensemble firing frequency, even if individual ORN contributions might already be saturated. Combinatorial coding in the sense of odor-specific vectors of frequency responses could not be evaluated as the ORN responses were normalized to their response at $80 \mu \mathrm{M}$.

\subsubsection{Role of glomeruli}

In $\mathrm{M} / \mathrm{T}$ cells of the olfactory bulb, odor identities and odor concentrations are well-predicted by patterns of their first-spike latencies, which are superior to patterns of their firing rate [23]. How are these latencies generated? For the odorant stimulation with methionine at $50 \mu \mathrm{M}$, consistent latency differences between $\mathrm{M} / \mathrm{T}$ cells up to $500 \mathrm{~ms}$ were detected, which cannot be easily explained by individual ORN latencies - the cell-specific contribution to their latency variation (compare standard deviations of $t_{0}-t_{\mathrm{m}}$ and $t_{0}-t_{\mathrm{r}}$ at $40 \mu \mathrm{M})$ is lower by a factor of about 5 .

Additional measurements at ORN axon terminals in olfactory glomeruli showed that the sum of ORN responses already exhibit the latency pattern observed in $\mathrm{M} / \mathrm{T}$ cell populations (with slightly less significance). The latencies in the olfactory bulb are thus generated by summation over ORN 
ensemble responses, which could be an additional feature of glomeruli in olfactory coding. For the observed concentrations, it is likely that M/T latencies are mainly determined by the combined AP firing rates of individual ORNs, essentially computing a transformation of olfactory information from frequency to latency space.

\subsection{Calcium channel microdomains and func- tion of BK channels}

Using a fast sequential line scanning mode at $1250 \mathrm{f} / \mathrm{s}$, it was possible to detect $\mathrm{Ca}^{2+}$ influx in single ORNs. As the fluorescence signal during depolarization steps was mostly dynamic (in contrast to steady-state situations described in [43]), influx regions were characterized not by the absolute fluorescent amplitude, but by the rate of fluorescent increase, together with the time constant describing saturation and decay after a pixel-wise fit. Through this method it was possible to increase image contrast in the detection of hotspots of $\mathrm{Ca}^{2+}$ influx.

$\mathrm{Ca}^{2+}$ microdomains were further investigated in our lab by Dr. Guobin Bao (Dep. of Neurophysiology and Cellular Biophysics, University of Göttingen) [2]. Here, areas of $\mathrm{Ca}^{2+}$ influx imaged at slower frame rates could be colocalized with BK channels that were imaged using Alexa-488 labeled iberiotoxin. As the main role of BK channels, fast repolarization and thus facilitation of high ORN firing rates could be identified, as the maximum AP frequency was reduced by blocking BK channels with iberiotoxin.

The reduction in response amplitudes measured in Section 3.3, where several ORNs were exposed to the same concentration of iberiotoxin, support these findings in the sense that the BK channel can positively modulate the response to odors. Only a subset of ORNs showed amplitude reductions upon BK inhibition, possibly meaning that BK channels are not expressed in all ORNs at the same level, or that the unaffected ORNs responded at too low AP frequencies to be modulated. 


\section{Chapter 5}

\section{Summary}

Odor space is large. This is one of the main problems the olfactory system has to solve, and it solves it well. Its strategy in detecting and discriminating a multitude of odors with a limited number of neurons lies in the so-called combinatorial coding. By having a set of olfactory receptor neurons (ORN), each with a broad but specific sensitivity to different odors, their response pattern can be used to efficiently recognize many odors. Recent findings suggest that response patterns are not necessarily static, but carry information in the time domain. In the olfactory bulb (OB), odor identity was found to be better described by latency patterns than by firing rates.

Motivated by these discoveries, this thesis aimed at the investigation of ORN population responses in how they are able to generate the latency code observable in the OB. For the measured odor concentration range, it was shown that latency coding is not dominant on the ORN level, and the main information is carried by ORN firing rates. Olfactory glomeruli thus seem to play a role in converting the set of ORN firing rates to a latency. Supplementing the population measurements, $\mathrm{Ca}^{2+}$ influx through voltagegated $\mathrm{Ca}^{2+}$ channels (VGCC) in single ORNs was imaged at high frame rates, discovering clustering of VGCCs. 


\section{Chapter 6}

\section{Bibliography}

[1] N. M. Abraham, H. Spors, A. Carleton, T. W. Margrie, T. Kuner, and A. T. Schaefer. Maintaining accuracy at the expense of speed: stimulus similarity defines odor discrimination time in mice. Neuron, 44(5):865876, Dec 2004.

[2] G. Bao. Detection and functional analysis of $\mathrm{Ca}^{2}+$ microdomains and BK channels in olfactory receptor neurons of larval Xenopus laevis. Phdthesis, University of Göttingen, Göttingen, August 2010.

[3] D. A. Berkowicz, P. Q. Trombley, and G. M. Shepherd. Evidence for glutamate as the olfactory receptor cell neurotransmitter. J Neurophysiol, 71(6):2557-2561, Jun 1994.

[4] L. Buck and R. Axel. A novel multigene family may encode odorant receptors: a molecular basis for odor recognition. Cell, 65(1):175-187, Apr 1991.

[5] S. Candia, M. L. Garcia, and R. Latorre. Mode of action of iberiotoxin, a potent blocker of the large conductance ca $(2+)$-activated $\mathrm{k}+$ channel. Biophys J, 63(2):583-590, Aug 1992.

[6] R. M. Carey, J. V. Verhagen, D. W. Wesson, N. Pírez, and M. Wachowiak. Temporal structure of receptor neuron input to the olfactory 
bulb imaged in behaving rats. J Neurophysiol, 101(2):1073-1088, Feb 2009.

[7] C.-C. Chien, H.-H. Chen, S.-F. Lai, K.-C. Wu, X. Cai, Y. Hwu, C. Petibois, Y. Chu, and G. Margaritondo. Gold nanoparticles as highresolution x-ray imaging contrast agents for the analysis of tumor-related micro-vasculature. J Nanobiotechnology, 10:10, 2012.

[8] R. S. Dhallan, K.-W. Yau, K. A. Schrader, and R. R. Reed. Primary structure and functional expression of a cyclic nucleotide-activated channel from olfactory neurons. Nature, 347(6289):184-187, Sep 1990.

[9] K. B. Døving, K.-A. Hansson, T. Backström, and E. H. Hamdani. Visualizing a set of olfactory sensory neurons responding to a bile salt. $J$ Exp Biol, 214(Pt 1):80-87, Jan 2011.

[10] K. B. Døving, K. Sandvig, and A. Kasumyan. Ligand-specific induction of endocytosis in taste receptor cells. J Exp Biol, 212(Pt 1):42-49, Jan 2009.

[11] F. A. Edwards, A. Konnerth, B. Sakmann, and T. Takahashi. A thin slice preparation for patch clamp recordings from neurones of the mammalian central nervous system. Pflugers Arch, 414(5):600-612, Sep 1989.

[12] S. Firestein. How the olfactory system makes sense of scents. Nature, 413(6852):211-218, Sep 2001.

[13] R. A. Fisher. On the "probable error" of a coefficient of correlation deduced from a small sample. Metron, 1:3-32, 1921.

[14] K. R. Gee, K. A. Brown, W. N. Chen, J. Bishop-Stewart, D. Gray, and I. Johnson. Chemical and physiological characterization of fluo-4 ca(2+)-indicator dyes. Cell Calcium, 27(2):97-106, Feb 2000.

[15] T. V. Getchell. Functional properties of vertebrate olfactory receptor neurons. Physiol Rev, 66(3):772-818, Jul 1986. 
[16] A. S. Ghatpande and J. Reisert. Olfactory receptor neuron responses coding for rapid odour sampling. J Physiol, 589(Pt 9):2261-2273, May 2011.

[17] S. Gliem. Characterization of olfactory receptor gene expression in the olfactory epithelium of larval Xenopus laevis. Phdthesis, University of Göttingen, Göttingen, September 2010.

[18] G. H. Gold. Controversial issues in vertebrate olfactory transduction. Annu Rev Physiol, 61:857-871, 1999.

[19] J. F. Hainfeld, D. N. Slatkin, T. M. Focella, and H. M. Smilowitz. Gold nanoparticles: a new x-ray contrast agent. Br J Radiol, 79(939):248-253, Mar 2006.

[20] H. Hamana, J. Hirono, M. Kizumi, and T. Sato. Sensitivity-dependent hierarchical receptor codes for odors. Chem Senses, 28(2):87-104, Feb 2003.

[21] D. T. Jones and R. R. Reed. Golf: an olfactory neuron specific-g protein involved in odorant signal transduction. Science, 244(4906):790-795, May 1989.

[22] S. Junek, T.-W. Chen, M. Alevra, and D. Schild. Activity correlation imaging: visualizing function and structure of neuronal populations. Biophys J, 96(9):3801-3809, May 2009.

[23] S. Junek, E. Kludt, F. Wolf, and D. Schild. Olfactory coding with patterns of response latencies. Neuron, 67(5):872-884, Sep 2010.

[24] S. J. Kleene. Both external and internal calcium reduce the sensitivity of the olfactory cyclic-nucleotide-gated channel to camp. J Neurophysiol, 81(6):2675-2682, Jun 1999.

[25] J. Klingauf and E. Neher. Modeling buffered ca2 + diffusion near the membrane: implications for secretion in neuroendocrine cells. Biophys J, 72(2 Pt 1):674-690, Feb 1997. 
[26] C. D. Kuglin and D. C. Hines. The phase correlation image alignment method. Proc. Int. Conf. Cybernetics Society, page 163-165, 1975.

[27] T. Kurahashi and A. Menini. Mechanism of odorant adaptation in the olfactory receptor cell. Nature, 385(6618):725-729, Feb 1997.

[28] D. G. Laing. Identification of single dissimilar odors is achieved by humans with a single sniff. Physiol Behav, 37(1):163-170, 1986.

[29] F. Lang and P. Lang. Basiswissen Physiologie. Springer-Lehrbuch. Springer, 2007.

[30] B.-J. Lin, T.-W. Chen, and D. Schild. Cell type-specific relationships between spiking and [ca2+]i in neurons of the xenopus tadpole olfactory bulb. J Physiol, 582(Pt 1):163-175, Jul 2007.

[31] M. Ma and G. M. Shepherd. Functional mosaic organization of mouse olfactory receptor neurons. Proc Natl Acad Sci U S A, 97(23):1286912874, Nov 2000.

[32] L. P. Maguire, S. Szilagyi, and R. E. Scholten. High performance laser shutter using a hard disk drive voice-coil actuator. Review of Scientific Instruments, 75(9):3077-3079, 2004.

[33] B. Malnic, J. Hirono, T. Sato, and L. B. Buck. Combinatorial receptor codes for odors. Cell, 96(5):713-723, Mar 1999.

[34] I. Manzini. Diversity of transduction mechanisms in receptor neurons of the main olfactory epithelium in Xenopus laevis tadpoles. PhD thesis, Georg-August-University Göttingen, Göttingen, December 2002.

[35] I. Manzini, C. Brase, T.-W. Chen, and D. Schild. Response profiles to amino acid odorants of olfactory glomeruli in larval Xenopus laevis. $J$ Physiol, 581(2):567-579, Jun 2007.

[36] I. Manzini and S. Korsching. The peripheral olfactory system of vertebrates: molecular, structural and functional basics of the sense of smell. e-Neuroforum, 2:68-77, 2011. 
[37] I. Manzini, F. Peters, and D. Schild. Odorant responses of Xenopus laevis tadpole olfactory neurons: a comparison between preparations. $J$ Neurosci Methods, 121(2):159-167, Dec 2002.

[38] I. Manzini and D. Schild. Multidrug resistance transporters in the olfactory receptor neurons of Xenopus laevis tadpoles. J Physiol, 546(2):375385, Jan 2003.

[39] I. Manzini and D. Schild. Classes and narrowing selectivity of olfactory receptor neurons of Xenopus laevis tadpoles. J Gen Physiol, 123(2):99107, Feb 2004.

[40] I. Manzini, T.-S. Schweer, and D. Schild. Improved fluorescent (calcium indicator) dye uptake in brain slices by blocking multidrug resistance transporters. J Neurosci Methods, 167(2):140-147, January 2008.

[41] S. D. Munger. Olfaction: Noses within noses. Nature, 459(7246):521522, May 2009.

[42] S. D. Munger, T. Leinders-Zufall, and F. Zufall. Subsystem organization of the mammalian sense of smell. Annu Rev Physiol, 71:115-140, 2009.

[43] M. Naraghi and E. Neher. Linearized buffered ca2+ diffusion in microdomains and its implications for calculation of $[\mathrm{ca} 2+]$ at the mouth of a calcium channel. J Neurosci, 17(18):6961-6973, Sep 1997.

[44] P. D. Nieuwkoop and J. Faber, editors. Normal Table of Xenopus laevis (Daudin). Garland Publishing, June 1994.

[45] Y. Niimura and M. Nei. Evolutionary changes of the number of olfactory receptor genes in the human and mouse lineages. Gene, 346:23-28, Feb 2005 .

[46] Y. Oka, Y. Takai, and K. Touhara. Nasal airflow rate affects the sensitivity and pattern of glomerular odorant responses in the mouse olfactory bulb. J Neurosci, 29(39):12070-12078, Sep 2009. 
[47] M. Omura, H. Sekine, T. Shimizu, H. Kataoka, and K. Touhara. In situ $\mathrm{Ca}^{2+}$ imaging of odor responses in a coronal olfactory epithelium slice. Neuroreport, 14(8):1123-1127, Jun 2003.

[48] S. Pifferi, A. Boccaccio, and A. Menini. Cyclic nucleotide-gated ion channels in sensory transduction. FEBS Lett, 580(12):2853-2859, May 2006.

[49] N. Rawson, J. Eberwine, R. Dotson, J. Jackson, P. Ulrich, and D. Restrepo. Expression of mRNAs encoding for two different olfactory receptors in a subset of olfactory receptor neurons. J Neurochem, 75(1):185195, Jul 2000.

[50] J. Reisert and H. R. Matthews. Response properties of isolated mouse olfactory receptor cells. J Physiol, 530(Pt 1):113-122, Jan 2001.

[51] J. Reisert and H. R. Matthews. Responses to prolonged odour stimulation in frog olfactory receptor cells. J Physiol, 534(Pt 1):179-191, Jul 2001.

[52] K. J. Ressler, S. L. Sullivan, and L. B. Buck. A zonal organization of odorant receptor gene expression in the olfactory epithelium. Cell, 73(3):597-609, May 1993.

[53] K. J. Ressler, S. L. Sullivan, and L. B. Buck. Information coding in the olfactory system: evidence for a stereotyped and highly organized epitope map in the olfactory bulb. Cell, 79(7):1245-1255, Dec 1994.

[54] D. Rinberg, A. Koulakov, and A. Gelperin. Sparse odor coding in awake behaving mice. J Neurosci, 26(34):8857-8865, Aug 2006.

[55] J.-P. Rospars, P. Lánský, A. Duchamp, and P. Duchamp-Viret. Relation between stimulus and response in frog olfactory receptor neurons in vivo. Eur J Neurosci, 18(5):1135-1154, Sep 2003.

[56] J. P. Rospars, L. P., P. Duchamp-Viret, and A. Duchamp. Spiking frequency versus odorant concentration in olfactory receptor neurons. Biosystems, 58(1-3):133-141, 2000. 
[57] L. Salkoff, A. Butler, G. Ferreira, C. Santi, and A. Wei. Highconductance potassium channels of the slo family. Nat Rev Neurosci, 7(12):921-931, Dec 2006.

[58] D. Schild. A computer-controlled device for the application of odours to aquatic animals. J Electrophysiol Techn., 12:71-79, 1985.

[59] D. Schild. Whole-cell currents in olfactory receptor cells of Xenopus laevis. Exp Brain Res, 78(2):223-232, 1989.

[60] D. Schild, A. Gennerich, and H. A. Schultens. Microcontrollers as inexpensive pulse generators and parallel processors in electrophysiological experiments. Med Biol Eng Comput, 34(4):305-307, Jul 1996.

[61] D. Schild, A. Jung, and H. A. Schultens. Localization of calcium entry through calcium channels in olfactory receptor neurones using a laser scanning microscope and the calcium indicator dyes Fluo-3 and FuraRed. Cell Calcium, 15(5):341-348, May 1994.

[62] D. Schild and D. Restrepo. Transduction mechanisms in vertebrate olfactory receptor cells. Physiol Rev, 78(2):429-466, Apr 1998.

[63] J. E. Schwob. Neural regeneration and the peripheral olfactory system. Anat Rec, 269(1):33-49, Feb 2002.

[64] B. Slotnick. Odor-sampling time of mice under different conditions. Chem Senses, 32(5):445-454, Jun 2007.

[65] G. D. Smith. Analytical steady-state solution to the rapid buffering approximation near an open ca2+ channel. Biophys J, 71(6):3064-3072, Dec 1996.

[66] H. Spors, M. Wachowiak, L. B. Cohen, and R. W. Friedrich. Temporal dynamics and latency patterns of receptor neuron input to the olfactory bulb. J Neurosci, 26(4):1247-1259, Jan 2006.

[67] A. B. Stephan, E. Y. Shum, S. Hirsh, K. D. Cygnar, J. Reisert, and H. Zhao. ANO2 is the cilial calcium-activated chloride channel that 
may mediate olfactory amplification. Proc Natl Acad Sci U S A, 106(28):11776-11781, Jul 2009.

[68] H. Tian and M. Ma. Activity plays a role in eliminating olfactory sensory neurons expressing multiple odorant receptors in the mouse septal organ. Mol Cell Neurosci, 38(4):484-488, Aug 2008.

[69] N. Uchida and Z. F. Mainen. Speed and accuracy of olfactory discrimination in the rat. Nat Neurosci, 6(11):1224-1229, Nov 2003.

[70] K. Ukhanov, Y. Bobkov, and B. W. Ache. Imaging ensemble activity in arthropod olfactory receptor neurons in situ. Cell Calcium, 49(2):100107, Feb 2011.

[71] R. Vassar, S. K. Chao, R. Sitcheran, J. M. Nuñez, L. B. Vosshall, and R. Axel. Topographic organization of sensory projections to the olfactory bulb. Cell, 79(6):981-991, Dec 1994.

[72] D. W. Wesson, R. M. Carey, J. V. Verhagen, and M. Wachowiak. Rapid encoding and perception of novel odors in the rat. PLoS Biol, 6(4):e82, Apr 2008.

[73] Y. C. Wu, T. Tucker, and R. Fettiplace. A theoretical study of calcium microdomains in turtle hair cells. Biophys J, 71(5):2256-2275, Nov 1996.

[74] J. M. Young, C. Friedman, E. M. Williams, J. A. Ross, L. TonnesPriddy, and B. J. Trask. Different evolutionary processes shaped the mouse and human olfactory receptor gene families. Hum Mol Genet, 11(5):535-546, Mar 2002.

[75] D. Zenisek, V. Davila, L. Wan, and W. Almers. Imaging calcium entry sites and ribbon structures in two presynaptic cells. J Neurosci, 23(7):2538-2548, Apr 2003.

[76] X. Zhang and S. Firestein. The olfactory receptor gene superfamily of the mouse. Nat Neurosci, 5(2):124-133, Feb 2002. 


\section{Acknowledgements}

First of all, I would like to thank my supervisor Prof. Dr. Dr. Schild for giving me the opportunity to work in his lab, his helpful advice and discussion, and the great freedom offered in following my ideas.

Many thanks to Prof. Dr. Salditt and Prof. Dr. Schmidt for being in my thesis committee and giving me advice.

I am greatful to Dr. Stephan Junek for the nice time constructing the LIM, the discussions, and the friendship.

I want to thank Alexander Brinkmann and Sebastian Gliem for proofreading of the manuscript in the last minute.

And thanks to all friends and collegues from the lab for the creative and friendly atmoshpere, ranging from coffee sessions, scientific debates, to strange philosophy. 


\title{
Curriculum vitæ
}

\author{
Personal \\ Name Mihai Alevra \\ Date of birth April $29^{\text {th }} 1978$ \\ Place of birth Bukarest, Romania \\ E-mail malevra@gwdg.de
}

\section{Education}

since 2/2009 PhD in the laboratory of Prof. Dr. Dr. Schild, Dep. of Neurophysiology and Cellular Biophysics

Enrolled at the International Max Planck Research School "Physics of Biological and Complex Systems", Göttingen Graduate School for Neurosciences and Molecular Biosciences

3/2008 Diploma in physics, "Biophysikalische Charakterisierung schneller Signalprozesse in olfaktorischen Rezeptorneuronen mit einem konfokalen Linienrastermikroskop" 